\title{
Constraints on $\mu$-distortion fluctuations and primordial non-Gaussianity from Planck data
}

\author{
Rishi Khatri, ${ }^{a}$ Rashid Sunyaev ${ }^{a, b, c}$ \\ ${ }^{a}$ Max Planck Institut für Astrophysik \\ , Karl-Schwarzschild-Str. 1 85741, Garching, Germany \\ ${ }^{b}$ Space Research Institute, Russian Academy of Sciences, Profsoyuznaya 84/32, 117997 Moscow, \\ Russia \\ ${ }^{c}$ Institute for Advanced Study, Einstein Drive, Princeton, New Jersey 08540, USA \\ E-mail: khatri@mpa-garching.mpg.de
}

\begin{abstract}
We use the Planck HFI channel maps to make an all sky map of $\mu$-distortion fluctuations. Our $\mu$-type distortion map is dominated by the $y$-type distortion contamination from the hot gas in the low redshift Universe and we can thus only place upper limits on the $\mu$-type distortion fluctuations. For the amplitude of $\mu$-type distortions on $10^{\prime}$ scales we get the limit on root mean square (rms) value $\mu_{\mathrm{rms}}^{10^{\prime}}<6.4 \times 10^{-6}$, a limit 14 times stronger than the COBE-FIRAS (95\% confidence) limit on the mean of $\langle\mu\rangle<90 \times 10^{-6}$. Using our maps we also place strong upper limits on the auto angular power spectrum of $\mu, C_{\ell}^{\mu \mu}$ and the cross angular power spectrum of $\mu$ with the CMB temperature anisotropies, $C_{\ell}^{\mu T}$. The strongest observational limits are on the largest scales, $\ell(\ell+1) /\left.(2 \pi) C_{\ell}^{\mu \mu}\right|_{\ell=2-26}<(2.3 \pm$ $1.0) \times 10^{-12}$ and $\ell(\ell+1) /\left.(2 \pi) C_{\ell}^{\mu T}\right|_{\ell=2-26}<(2.6 \pm 2.6) \times 10^{-12} \mathrm{~K}$. Our observational limits can be used to constrain new physics which can create spatially varying energy release in the early Universe between redshifts $5 \times 10^{4} \lesssim z \lesssim 2 \times 10^{6}$. We specifically apply our observational results to constrain the primordial non-Gaussianity of the local type, when the source of $\mu$-distortion is Silk damping, for very squeezed configurations with the wavenumber for the short wavelength mode $46 \lesssim k_{\mathrm{S}} \lesssim 10^{4} \mathrm{Mpc}^{-1}$ and for the long wavelength mode $k_{\mathrm{L}} \approx 10^{-3} \mathrm{Mpc}^{-1}$. Our limits on the primordial non-Gaussianity parameters are $f_{\mathrm{NL}}<10^{5}, \tau_{\mathrm{NL}}<1.4 \times 10^{11}$ for $k_{\mathrm{S}} / k_{\mathrm{L}} \approx 5 \times 10^{4}-10^{7}$. We also give a new derivation of the evolution of the $\mu$-distortion fluctuations through the $y$-distortion era and the recombination epoch until today resulting in very simple expressions for the cross and auto power spectra in the squeezed limit. We also introduce mixing of Bose-Einstein spectra due to Silk damping and $y^{\mathrm{BE}}$-type distortions. The $\mu$-type distortion map and masks are now publicly available.
\end{abstract}

Keywords: cosmic background radiation, cosmology:theory, early universe 


\section{Contents}

1 Introduction 1

$2 \mu$-type distortions in Planck channels $\quad 3$

3 A $\mu$-type distortion map from Planck HFI data 4

4 Constraints on the $\mu$-distortion fluctuations $\quad 6$

4.1 Amplitude of fluctuations 6

$\begin{array}{lll}4.2 & \text { Auto and cross power spectrum } & 7\end{array}$

5 Theory of fluctuations of $\mu$-type distortions from Silk damping 11

5.1 The auto and cross power spectrum of $\mu$-distortion fluctuations 15

5.2 Constraints on primordial non-Gaussianity from $\mu$-distortion fluctuations 17

6 Conclusions

\section{Introduction}

Complete thermodynamic equilibrium between the photons and the electromagnetic plasma exists only in the very early Universe in the standard cosmological model. The memory of any event which injects energy into the electromagnetic plasma is quickly erased under such circumstances, apart from the change in the temperature of the plasma and the photons. This fundamental limit on how far and how well we can probe the early Universe using the CMB photons is much further than the last scattering surface at $z \sim 1100[1-3]$ that we observe with the CMB anisotropy experiments such as COBE-DMR (Cosmic Background Explorer - Differential Microwave Radiometer) [4], WMAP (Wilkinson Microwave Anisotropy Probe) [5], Planck [6], SPT (South Pole Telescope) [7], ACT (Atacama Cosmology Telescope) [8] and many others. ${ }^{1}$

An important landmark across which the properties of the Universe change in a significant way in a short time is the blackbody surface $[9,10]$ at $z \approx 2 \times 10^{6}$. At times earlier than this landmark, $z \gtrsim 2 \times 10^{6}$, the photon creation at low frequencies $(x \equiv v / T \ll 1$, where $v$ is the photon frequency, $T$ is the temperature of the electromagnetic plasma, $x$ is the dimensionless frequency and we are using natural units with Planck's constant $(h)$ and Boltzmann constant $\left(k_{\mathrm{B}}\right)$ equal to unity.) combined with the redistribution of photons over the whole spectrum by Compton scattering maintains a Planck spectrum for the photons [9]. The dominant process for the photon creation is the double Compton scattering for a low baryon density Universe such as ours [11] with a small contribution from bremsstrahlung. At lower redshifts, $z \lesssim 2 \times 10^{6}$, the photon creation by double Compton and bremsstrahlung becomes inefficient, making the photon number a conserved quantity. Any injection of energy is still redistributed by Compton scattering but the inability to create and destroy photons means that the equilibrium spectrum is not the Planck spectrum but the Bose-Einstein spectrum (see e.g. [12]) with a dimensionless chemical potential $\left(\mu \equiv-\mu_{E} / T\right.$, where $\mu_{E}$ is the thermodynamic

\footnotetext{
${ }^{1}$ See http://http://lambda.gsfc.nasa.gov/links/experimental_sites.cfm for a complete list of CMB experiments
} 
chemical potential) that does not vanish in general,

$$
n_{\mathrm{BE}}(x)=\frac{1}{e^{x+\mu}-1} .
$$

The Bose-Einstein spectrum is a stationary solution of the Kompaneets equation [9, 13].

For observational purpose and also for comparison with the $y$-type distortions [14] it is convenient to define the dimensionless frequency not with respect to the temperature of the Bose-Einstein spectrum but with respect to the temperature of a Planck spectrum which has the same number density of photons as the Bose-Einstein spectrum, $N_{\mathrm{BE}}(T, \mu) \equiv N_{\mathrm{Pl}}\left(T_{\text {ref }}\right) \Rightarrow\left(T-T_{\text {ref }}\right) / T_{\text {ref }} \approx 0.456 \mu, x_{\text {ref }} \equiv$ $v / T_{\text {ref. }}$ In terms of this reference temperature we have

$$
\begin{aligned}
n_{\mathrm{BE}}\left(x_{\mathrm{ref}}\right) & \equiv \frac{1}{e^{x_{\mathrm{ref}} T_{\mathrm{ref}} / T+\mu}-1} \\
& \stackrel{\mu \ll 1}{\approx} \frac{1}{e^{x_{\mathrm{ref}}}-1}+\frac{\mu e_{\mathrm{ref}}^{x}}{\left(e^{x_{\mathrm{ref}}}-1\right)^{2}}\left(\frac{x_{\mathrm{ref}}}{2.19}-1\right) \\
& \equiv n_{\mathrm{pl}}+\mu n_{\mu},
\end{aligned}
$$

where the first term is the Planck spectrum and the second term defines the $\mu$-type distortion in the limit of small distortions [9]. Note that at linear order, for small $\mu$, we have $\mu \equiv-\mu_{E} / T \approx-\mu_{E} / T_{\text {ref. }}$. We should emphasize that this re-definition is just for convenience of visualization and does not affect the analysis of data. This is because the monopole temperature of the CMB is also uncertain, at the sensitivity we will be working, and we must fit for the reference temperature simultaneously giving us the freedom to choose the reference temperature. We will use the definition $n_{\mu}$ from Eq. 1.2 throughout the rest of the paper with $T=2.725 \mathrm{~K}$ as the reference temperature dropping the subscript ref.

The $\mu$-type distortions thus probe the era that lies behind the last scattering surface and are sensitive to any process that injects energy into the electromagnetic plasma [see e.g. 15, 16] between redshifts $5 \times 10^{4} \lesssim z \lesssim 2 \times 10^{6}$ [17]. Most processes, owing to the statistical homogeneity of the Universe, give distortions which are identical in different regions of the Universe. Planck is insensitive to the absolute brightness of the sky and therefore cannot detect the constant or invariant component of the $\mu$-type distortions. One of the exceptions is the dissipation of primordial perturbations or sound waves in the electromagnetic plasma (Silk damping) [18-20] before recombination. The primordial perturbations excite standing sound waves in the tightly coupled baryon-photon fluid as they enter the horizon $[3,19,21]$. The mean free path of the photons for Thomson scattering on free electrons is very small during this time, they can however still diffuse to much larger scales doing a random walk in the sea of electrons. The (comoving) diffusion scale $\left(\lambda_{\mathrm{D}}\right)$ and wavenumber $\left(k_{\mathrm{D}}\right)$ are given during the radiation dominated era by (including both thermal conductivity and radiative viscosity) [18-20]

$$
\begin{aligned}
\lambda_{\mathrm{D}}=\frac{2 \pi}{k_{\mathrm{D}}} & =\left[\int_{0}^{\eta} \mathrm{d} \eta \frac{2 \pi c(1+z)}{6(1+R) n_{\mathrm{e}} \sigma_{\mathrm{T}}}\left(\frac{R^{2}}{1+R}+\frac{16}{15}\right)\right]^{1 / 2} \\
& \approx\left(\frac{16 \pi c}{135 \Omega_{\mathrm{r}}{ }^{1 / 2} H(0) n_{\mathrm{e}}(0) \sigma_{\mathrm{T}}(1+z)^{3}}\right)^{1 / 2},
\end{aligned}
$$

where $R \equiv \frac{3 \rho_{b}}{4 \rho_{\gamma}}, \rho_{b}$ is the baryon energy density, $\rho_{\gamma}$ is the photon energy density, $\eta$ is conformal time, $\sigma_{\mathrm{T}}$ is the Thomson cross section, $n_{\mathrm{e}}(\eta)$ is the electron number density, $H(\eta)$ is the Hubble parameter, $\Omega_{\mathrm{r}}$ is the radiation energy density in units of critical density today, $\eta=0$ is present time and $c$ is the speed of light. The diffusion or dissipation scale at $z=5 \times 10^{4}$ is $k_{\mathrm{D}} \approx 46 \mathrm{Mpc}^{-1}, \lambda_{\mathrm{D}} \approx 0.14 \mathrm{Mpc}$ 
and at $z=2 \times 10^{6}$ it is $k_{\mathrm{D}} \approx 1.1 \times 10^{4} \mathrm{Mpc}^{-1}, \lambda_{\mathrm{D}} \approx 0.55 \mathrm{kpc}$. The photon diffusion erases the perturbations on the diffusion scales, moving the energy in the perturbations or sound waves into the local CMB monopole spectrum, giving a chemical potential to the spectrum [9, 22-25]. If the primordial curvature perturbations are Gaussian, there is same power on the small scales $46 \lesssim k_{\mathrm{D}} \lesssim$ $10^{4} \mathrm{Mpc}^{-1}$ in different regions of the Universe, apart from the cosmic variance $\propto N_{\text {modes }}^{1 / 2}=\left(k_{\mathrm{L}} / k_{D}\right)^{3 / 2}$, where $k_{\mathrm{L}} \lesssim 0.01 \mathrm{Mpc}^{-1}$ is the separation of different regions in the Universe over which we wish to study the fluctuations. If the primordial non-Gaussianity of local type is present on these scales [26], the small scale power is correlated with the large scale fluctuations and the $\mu$-distortion has large scale anisotropy on the sky with amplitude dependent on the amplitude of the primordial non-Gaussianity. The fluctuations in the $\mu$-type distortions, and hence the primordial non-Gaussianity on these scales, is already accessible with Planck, which has much higher sensitivity compared to the COBE-FIRAS for a fluctuating signal [27].

We will use the component separation method LIL (Linearized Iterative Least-squares) [28] to separate the $\mu$-distortion component from Planck data. The small number of channels and similarity of the $\mu$-distortion spectrum with the $y$-type spectrum means that our map would be dominated by contamination from the low redshift $y$-type distortions and we will only be able to put upper limits on the $\mu$-distortion fluctuations and primordial non-Gaussianity. In principle, there is additional information in the intermediate-type distortions [17, 29-31] which is however inaccessible with Planck owing to the limited number of frequency channels. The maps and masks, created by one of us (RK), are made publicly available at http://www.mpa-garching.mpg.de/ khatri/muresults/.

\section{$2 \mu$-type distortions in Planck channels}

We will be using the 4 HFI channels of the Planck $(100,143,217,353 \mathrm{GHz})$. Each of these channels has finite bandwidth and we must integrate the intensity spectrum of $\mu$-type distortions $\left(2 h v^{3} / c^{2} n_{\mu}\right)$ over each spectral band. In addition it is also convenient to convert the intensity into thermodynamic $\mathrm{CMB}$ units defined by relating the change in intensity in the spectral band, $\Delta I_{v}$, to the change in the intensity $I_{\mathrm{pl}}(T)$ of the Planck spectrum for a change in temperature $\Delta T_{\mathrm{K}_{\mathrm{CMB}}}$,

$$
\begin{aligned}
\Delta I_{v} & =\Delta T_{\mathrm{K}_{\mathrm{CMB}}} \frac{\partial I_{\mathrm{pl}}}{\partial T} \\
& =\Delta T_{\mathrm{K}_{\mathrm{CMB}}} \frac{2 h v^{3}}{T c^{2}} \frac{x e^{x}}{\left(e^{x}-1\right)^{2}}
\end{aligned}
$$

The units of the maps released by Planck are $\mathrm{K}_{\mathrm{CMB}}$ for the 4 HFI channels we will be using. To convert any other spectrum, $n(x)$, to the CMB temperature units we should integrate both the intensity of the spectrum of interest and differential Planck spectrum over the transmission profile of the channels $w(v)[32]$ which are available on the Planck legacy archive,

$$
\Delta T_{\mathrm{K}_{\mathrm{CMB}}}=\frac{\int w(v) 2 h v^{3} / c^{2} n(v) \mathrm{d} v}{\int w(v) \partial I_{\mathrm{pl}} / \partial T \mathrm{~d} v} .
$$

For the $\mu$ distortion $n(x)=n_{\mu}$, and similarly for the $y$-type distortion we substitute $n(x)=n_{\mathrm{y}}$ where $n_{\mathrm{y}}$ is the $y$-type spectrum [14]

$$
n_{\mathrm{y}}=\frac{x e^{x}}{\left(e^{x}-1\right)^{2}}\left[x\left(\frac{e^{x}+1}{e^{x}-1}\right)-4\right]
$$




\begin{tabular}{|c|c|c|c|c|c|c|c|c|c|}
\hline Channel-GHz & 30 & 44 & 70 & 100 & 143 & 217 & 353 & 545 & 857 \\
\hline$y$ to $\mathrm{K}_{\mathrm{CMB}}$ & -5.3337 & -5.1752 & -4.7503 & -4.0309 & -2.7823 & 0.1941 & 6.2065 & 14.453 & 26.332 \\
$\mu$ to $\mathrm{K}_{\mathrm{CMB}}$ & -4.1611 & -2.2528 & -0.9414 & -0.2767 & 0.1606 & 0.5411 & 0.8053 & 0.9484 & 1.0413 \\
\hline
\end{tabular}

Table 1. Conversion factors from $y$ and $\mu$ to $\mathrm{K}_{\mathrm{CMB}}$. We have checked that the small difference in the $y$ conversion factors with respect to the [32] are due to the change in the transmission profile between the first and the second Planck data releases. We have used the later release to calculate the conversion factors. All values are for the full channels.

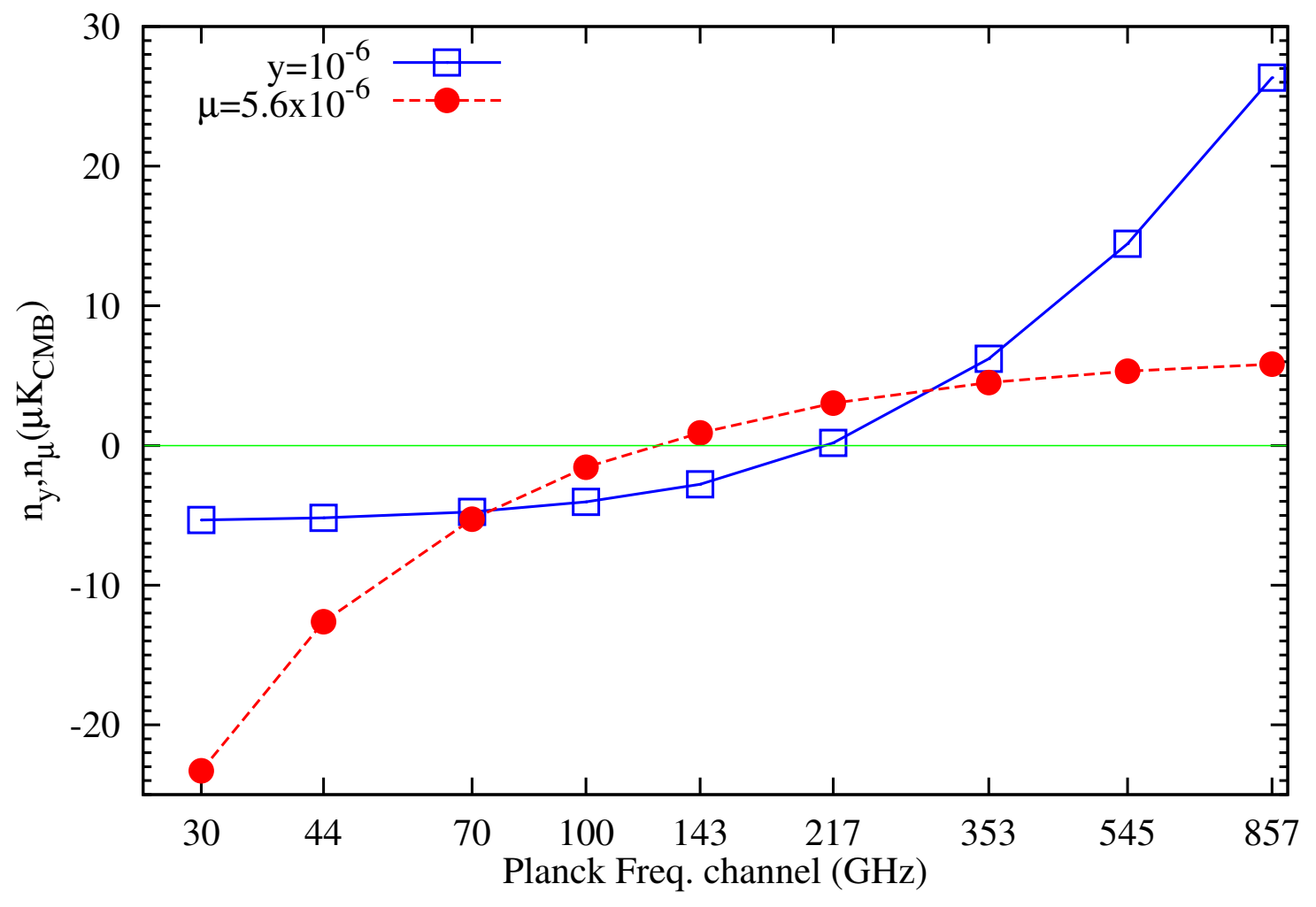

Figure 1. The spectra of $y$-type and $\mu$-type distortions in the $\mu \mathrm{K}_{\mathrm{CMB}}$ units as seen by Planck channels. We will only use the $4 \mathrm{HFI}$ channels, $100 \mathrm{GHz}$ to $353 \mathrm{GHz}$, in our analysis.

We give the conversion factors calculated for all Planck channels for both $\mu$ and $y$-type distortions in Table 1 and the spectra are plotted in Fig. 1. Our $y$ to $\mathrm{K}_{\mathrm{CMB}}$ conversion factors match those released by Planck [32] with a small difference because of our using the band transmission profile from the second data release. We will exploit the small difference in the two spectra to mask out as much of the $y$-distortion signal from galaxy clusters and groups as possible allowing us to put much stronger constraints on the $\mu$-type distortions compared to the constraints we would expect in the absence of such a mask.

\section{A $\mu$-type distortion map from Planck HFI data}

The component separation method, LIL, is described in [28] and its application to separate the $y$ type distortion from CMB and foregrounds is described in [33]. The algorithm to create the $\mu$-type distortion map is identical to that described in [33] with the $y$-type distortion spectrum replaced by 
the $\mu$-distortion spectrum given in Table 1 . We will only sketch the main features of the algorithm below and refer the reader to $[28,33]$ for details of the implementation.

LIL is a parameter fitting algorithm and we will fit a parametric model consisting of CMB + dust $+\mu$-distortion or CMB + dust $+y$-distortion to the 4 lowest Planck HFI channels at each pixel. The spectra of CMB and distortions are of course fixed and the only free parameters are the amplitudes. For the dust we fit a gray body dust model with temperature fixed to $18 \mathrm{~K}$ but allowing the spectral index $\beta_{\text {dust }}$ to vary between the limited range of $2<\beta_{\text {dust }}<3$ away from the galactic plane [see 28 , for exact definitions]. The constraint on the $\beta_{\text {dust }}$ means that the effective degrees of freedom is non-zero even though we fit 4 parameters to 4 data points. This is because away from the galactic plane, the main region of interest for us, the signal in $217 \mathrm{GHz}$ channel is too small to constrain $\beta_{\text {dust }}$ and we rarely reach a global minimum in $\chi^{2}$ in this parameter direction owing to the hard constraints imposed by us.

An advantage of the parameter fitting approach over the internal linear combination based methods [34-36] is that we get a quantitative goodness of fit estimate which tells us if the model being fit is the correct one. In particular, if we fit a $\mu$-type distortion spectrum to the pixels corresponding to clusters and groups of galaxies, we will get a $\chi^{2}$ which would be worse compared to the correct model with $y$-type distortion. We use this difference in $\chi^{2}$ between the two models to mask out clusters and groups according to the following algorithm

1. We mask a pixel if $\chi_{y}^{2}-\chi_{\mu}^{2}<-0.5$

2. We add the requirement that minimum hole size should be 10 pixels at HEALPix [37] resolution of nside $=2048$ i.e. if less than 10 contiguous pixels are getting masked then these pixels are unmasked.

3. We also mask all pixels with negative $y$-type distortion and $\chi_{y}^{2}>3.8$. This is signature of radio point sources.

4. We augment the resulting mask by additional 3 pixels

This simple algorithm masks most of the clusters and groups that are detected in our $y$-distortion map. In addition we explicitly mask $4.5^{\circ}$ radius regions around Virgo and Perseus, $1.5^{\circ}$ radius regions around all clusters with $S / N>30$ in the second Planck cluster catalog [38] and $0.75^{\circ}$ radius regions around all sources in the second Planck cluster catalog. We finally add the $86 \%$ $\mathrm{CO} /$ point source/galactic mask that was calculated in [33] and is publicly available at http://www . mpa-garching .mpg. de/ khatri/szresults/. Our final minimal mask for the $\mu$-distortion analysis, masking $25.8 \%$ of the sky, is shown in Fig. 2. We will augment this minimal mask using thresholds on the $545 \mathrm{GHz}$ channel map of Planck to select successively cleaner portions of the sky to test the robustness of our results to the dust contamination.

The probability distribution function (PDF) of the $\mu$-distortion map is shown in Fig. 3 for different sky fractions $f_{\text {sky }}$. The PDF is dominated by noise and residual $y$-distortion contamination at all sky fractions. For the $62 \%$ sky fraction there is also a small amount of contamination from the dust (since the masks were designed using the $545 \mathrm{GHz}$ map which is dominated by dust) which becomes negligible at $f_{\text {sky }}=45 \%$ and goes away completely at $f_{\text {sky }}=27 \%$. The contribution of the tails to the map variance and power spectrum is negligible. This is apparent immediately if we look at the same plot drawn on linear scale in Fig. 4. The sharp peak near the small values of $\mu$ is probably due to a small number of clean low noise pixels where the noise dominates over the contamination. The skewness towards positive values because of the residual $y$-distortion contamination is also apparent. 


\section{$74 \%$ minimal mask}

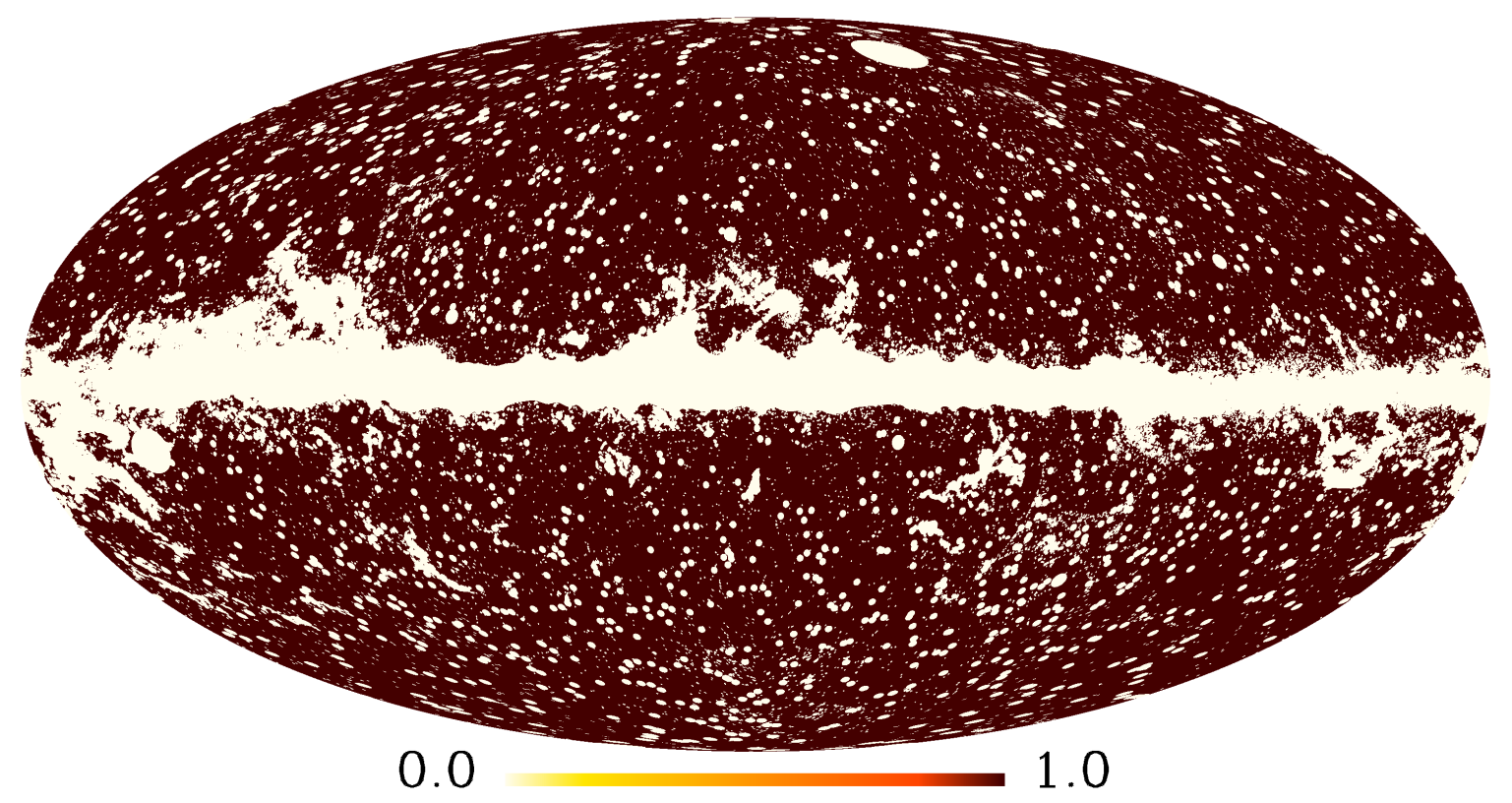

Figure 2. Minimal mask for $\mu$-distortion analysis masking clusters and groups of galaxies, point sources and galactic plane.

\section{Constraints on the $\mu$-distortion fluctuations}

\subsection{Amplitude of fluctuations}

The simplest constraint we can get on the $\mu$-distortion fluctuations is using the total power or variance of the map. We should in particular subtract the contribution of the noise. We make half ring $\mu$-maps from the Planck half-ring channel maps and calculate the half ring half difference (HRHD) $\mu$-map which gives us the white noise estimate in our full channel $\mu$-map. The total map variance $\sigma_{\text {map }}^{2}$ is sum of noise contribution, $\sigma_{\text {noise }}$ and signal $\mu_{\mathrm{rms}}^{2}$, assuming that the noise is uncorrelated with the signal,

$$
\sigma_{\text {map }}^{2}=\mu_{\text {rms }}^{2}+\sigma_{\text {noise }}^{2},
$$

where $\mu_{\mathrm{rms}}$ is the root mean squared value of the signal. We are in particular interested in the central variance, $\left(\mu_{\mathrm{rms}}^{c}\right)^{2} \equiv \mu_{\mathrm{rms}}^{2}-\langle\mu\rangle^{2}$, where the angular brackets indicate the ensemble average. The rms and mean values of $\mu$ from our map for different sky fractions are given in Table 2. Since our algorithm is non-linear, the half-ring half difference (HRHD) map underestimates the noise in the full channel map. A better, unbiased, estimate of the signal variance would be if we average the product of the two half-ring maps. The half-ring maps have uncorrelated white noise which automatically cancels in the cross-variance,

$$
\begin{aligned}
\left(\sigma_{\text {map }}^{X}\right)^{2} & \equiv\left\langle\mu^{\mathrm{hr} 1}(p) \mu^{\mathrm{hr} 2}(p)\right\rangle \\
& =\left(\mu_{\mathrm{rms}}^{X}\right)^{2},
\end{aligned}
$$




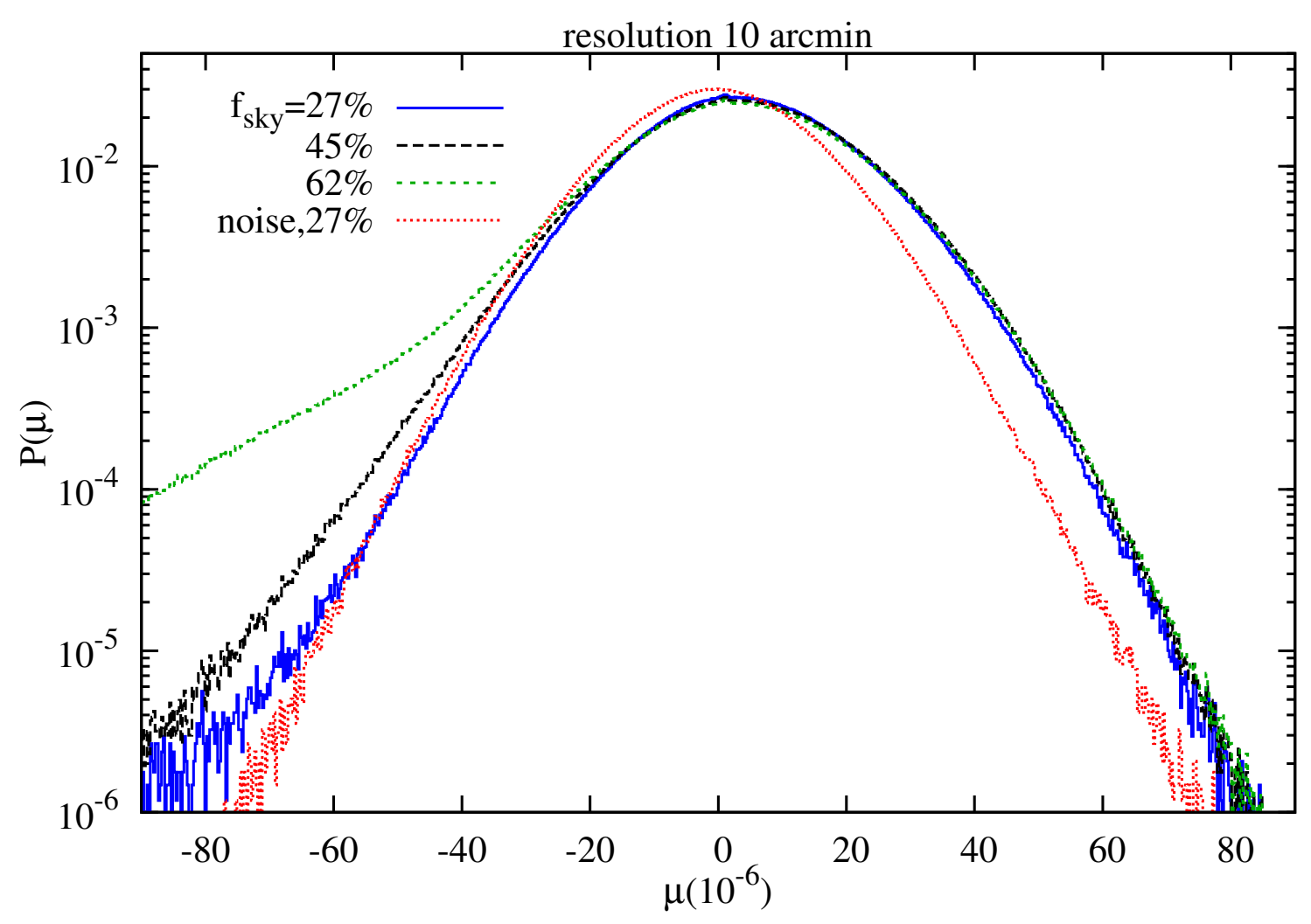

Figure 3. Probability distribution of $\mu$-distortion, $P(\mu)$, for different sky fractions, $f_{\text {sky }}$ along with the noise for $f_{\text {sky }}=27 \%$ at $10^{\prime}$ resolution calculated from the half-ring difference maps.

where $h r 1$ and $h r 2$ refer to the half-ring maps, the ensemble average is over all pixels $p$, and $X$ refers to the cross product of two maps. The central variance is then given by, $\left(\mu_{\mathrm{rms}}^{c X}\right)^{2} \equiv\left(\mu_{\mathrm{rms}}^{X}\right)^{2}-\langle\mu\rangle^{2}$. We will use $\mu_{\mathrm{rms}}^{c X}$ as our upper limit.

For our smallest mask $f_{\text {sky }}=70.7 \%$, the results are clearly dominated by the galactic contamination. The results however vary less rapidly from $f_{\text {sky }}=62.3 \%$ onward and seem to be converging with decreasing sky fractions. Assuming that all the signal is coming from the $y$-distortion contamination we get an upper limit of $\mu_{\mathrm{rms}}^{\text {central }}<6.1 \times 10^{-6}$. Note that since we are averaging over millions of pixels, even for the smallest sky fraction, the statistical error is negligible even after taking into account the fact that the noise in the neighboring pixels is correlated.

\subsection{Auto and cross power spectrum}

We can also calculate the angular power spectrum of our map, $C_{\ell}^{\mu \mu}$ and the cross power spectrum with the CMB temperature map, $C_{\ell}^{\mu T}$. For calculating the power spectra we use publicly available PolSpice code [39, 40] which deconvolves the effects of masks [41] in correlation space making it very fast. The code also calculates the covariance matrix [42-44] that we use to estimate the errors. We use large bins of $\Delta \ell=25$ to mitigate to some extent the effects of mode coupling due to our very complex mask. We augment our minimal mask using the $545 \mathrm{GHz}$ channel map smoothed to 2 degree FWHM beam to create the masks with successively cleaner regions of the sky. These masks were used in Table 2. For calculating the $C_{\ell}$ we apodize the mask with a Gaussian function in pixel space by replacing the $1 s$ in the mask by $1-\exp \left[-9 \theta^{2} / 2\left(\theta_{\mathrm{ap}}\right)^{2}\right]$ for $\theta<\theta_{\mathrm{ap}}=30^{\prime}$, where $\theta$ is the 


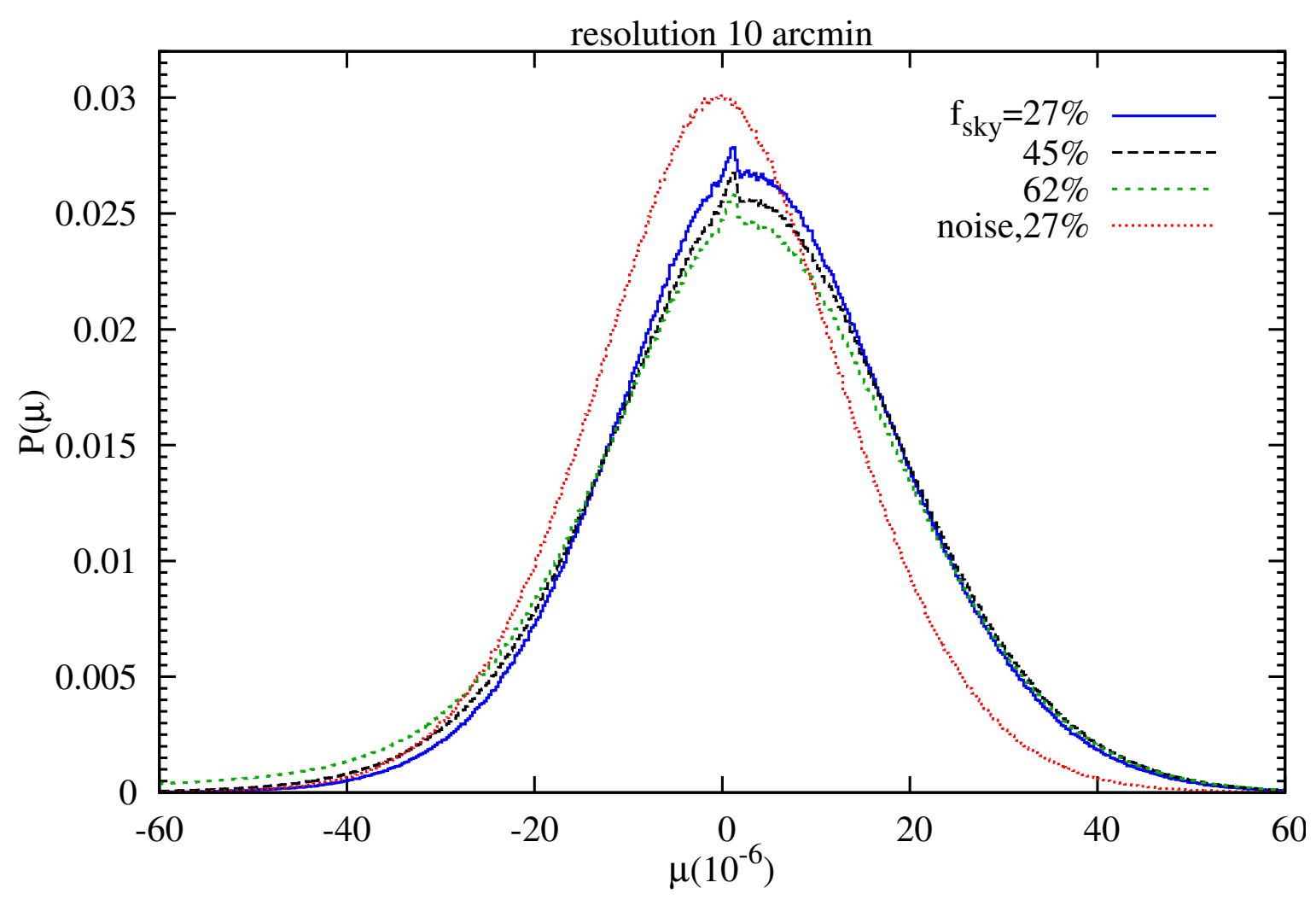

Figure 4. Same as Fig. 3 but with linear scale on the $y$ axes.

\begin{tabular}{|c|c|c|c|c|c|c|}
\hline$f_{\text {sky }}$ & $\begin{array}{c}\sigma_{\text {map }} \\
\left(\times 10^{-6}\right)\end{array}$ & $\begin{array}{c}\sigma_{\text {noise }} \\
\left(\times 10^{-6}\right)\end{array}$ & $\begin{array}{c}\langle\mu\rangle \\
\left(\times 10^{-6}\right)\end{array}$ & $\begin{array}{c}\mu_{\text {rms }} \\
\left(\times 10^{-6}\right)\end{array}$ & $\begin{array}{c}\mu_{\text {rms }}^{c} \\
\left(\times 10^{-6}\right)\end{array}$ & $\begin{array}{c}\mu_{\text {rms }}^{c X} \\
\left(\times 10^{-6}\right)\end{array}$ \\
\hline $70.7 \%$ & 24.23 & 18.00 & -0.40 & 16.21 & 16.21 & 9.81 \\
$62.3 \%$ & 18.67 & 15.69 & 1.87 & 10.13 & 9.95 & 7.35 \\
$54.0 \%$ & 17.40 & 15.15 & 2.85 & 8.57 & 8.08 & 6.77 \\
$45.1 \%$ & 16.80 & 14.77 & 3.48 & 8.01 & 7.21 & 6.51 \\
$36.6 \%$ & 16.43 & 14.48 & 3.77 & 7.77 & 6.79 & 6.37 \\
$26.9 \%$ & 15.95 & 14.14 & 3.70 & 7.38 & 6.39 & 6.10 \\
\hline
\end{tabular}

Table 2. Moments and other properties of the $\mu$-distortion map PDF for different sky fractions. The last column $\left(\mu_{\mathrm{rms}}^{c X}\right)$ is the limit on the $\mu$-distortion fluctuations calculated from cross-variance of half-ring maps while $\mu_{\mathrm{rms}}^{c}$ is calculated by subtracting noise estimate from half-ring half difference (HRHD) maps from the full channel map. The later slightly overestimates the signal variance.

distance of the pixel from the edge of the mask. To get an estimate of the power spectrum unbiased by noise we will use the cross-power spectrum of the half-ring maps automatically subtracting the uncorrelated noise. The auto power spectrum calculated in this way is shown in Fig. 5 for several sky fractions corrected for the $10^{\prime}$ beam of our maps in addition to the effect of the mask. There is a small decrease in the power on small scales when the sky fraction is reduced signifying the level of galactic contamination. On large scales, where we have the best constraints, the power spectra for different masks are consistent with each other (taking into account the error bars) and we will use the $f_{\text {sky }}=62.3 \%$ power spectrum on large scales to put limits on the $\mu$-distortion fluctuations and 


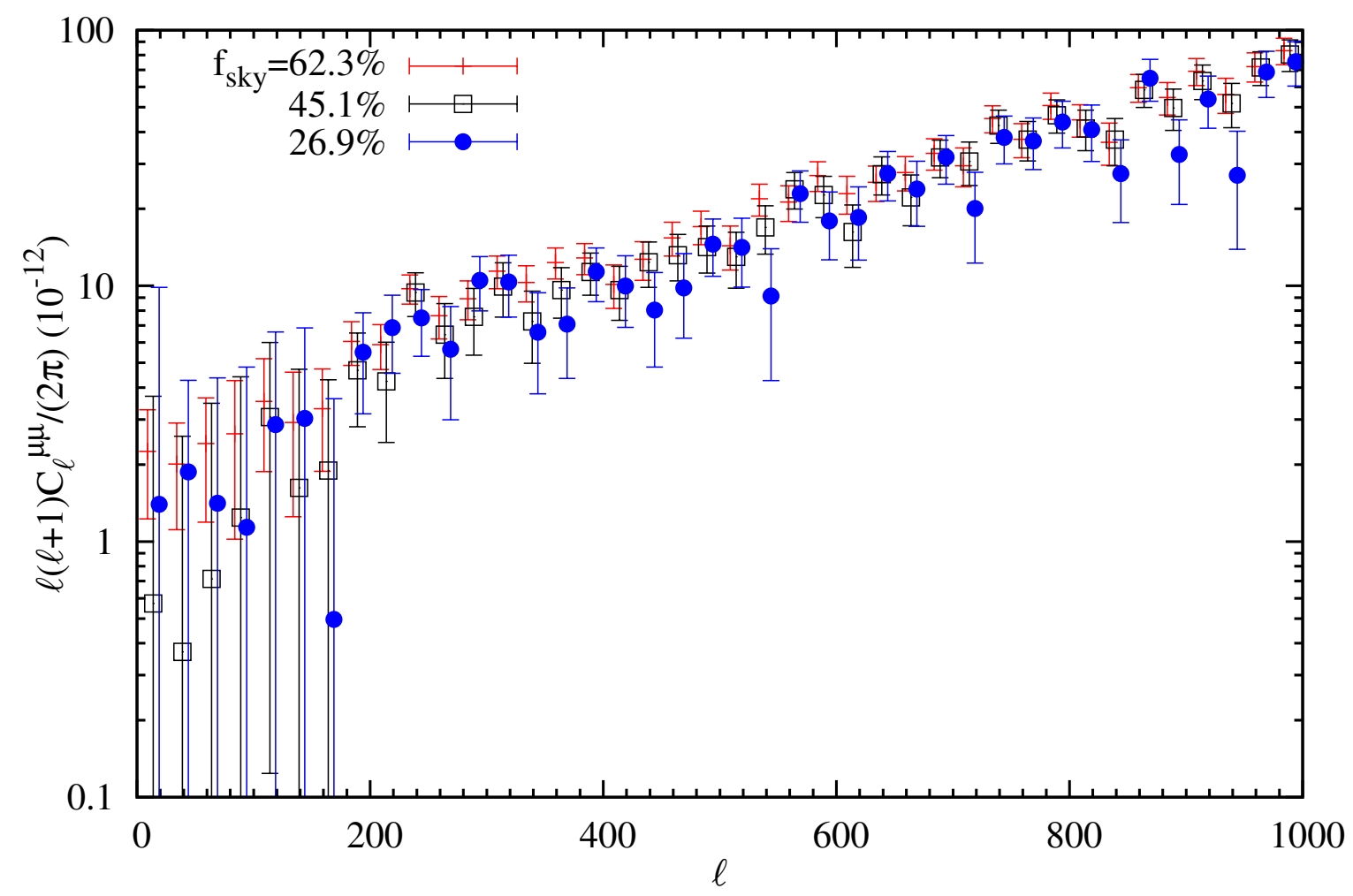

Figure 5. Auto power spectrum of $\mu$-type distortion fluctuations. The effect of the effective beam of $\mu$-map (10' FWHM) and mask has been corrected for. The points for different masks have been offset on the x-axes from the center of the $\Delta \ell=100$ bins to make them distinguishable.

primordial non-Gaussianity. Thus we have the limit on the power spectrum in the $\ell=2-26$ bin,

$$
\ell(\ell+1) C_{\ell}^{\mu \mu} /\left.(2 \pi)\right|_{\ell=2-26}<(2.3 \pm 1.0) \times 10^{-12}
$$

The cross spectrum of $\mu$ with primary CMB temperature anisotropies $T$ is given by

$$
\left\langle a_{\ell m}^{i} a_{\ell m}^{j}\right\rangle=C_{\ell}^{\mu T}+\left\langle n_{\ell m}^{i} n_{\ell m}^{j}\right\rangle
$$

where $i$ is the $\mu$-distortion map, either one of the half-ring maps or the full channel map. Similarly $j$ is one of the CMB temperature anisotropy maps and $n_{\ell m}^{i, j}$ is the noise in the respective map. As before we can choose the full mission maps and estimate and subtract the noise cross power spectrum estimated from the HRHD maps to get $=C_{\ell}^{\mu T}$ or we can use one of the half-ring $\mu$-distortion maps and the other half-ring map for $T$ in which case the cross noise term would vanish. We again expect the HRHD maps to give slightly biased estimates of the noise because of the non-linearity of our component separation algorithm. However on large scales, the cross-spectrum is dominated not by noise but the $\mathrm{CMB}$ anisotropies and we expect the effect of the noise to be sub-dominant. This can be seen in Fig. 6 where we have plotted the cross power spectra between our $\mu$-map and the SMICA (Spectral Matching Independent Component Analysis) CMB map released by the Planck collaboration [45] as well as between our $\mu$-map and the CMB map produced by LIL when fitting a $y+C M B+d u s t$ model [33], so that we have a CMB map where the $y$-type distortion signal is explicitly removed. All power spectra, except one labeled 'hr1x2', are calculated from the full channel maps and the noise 


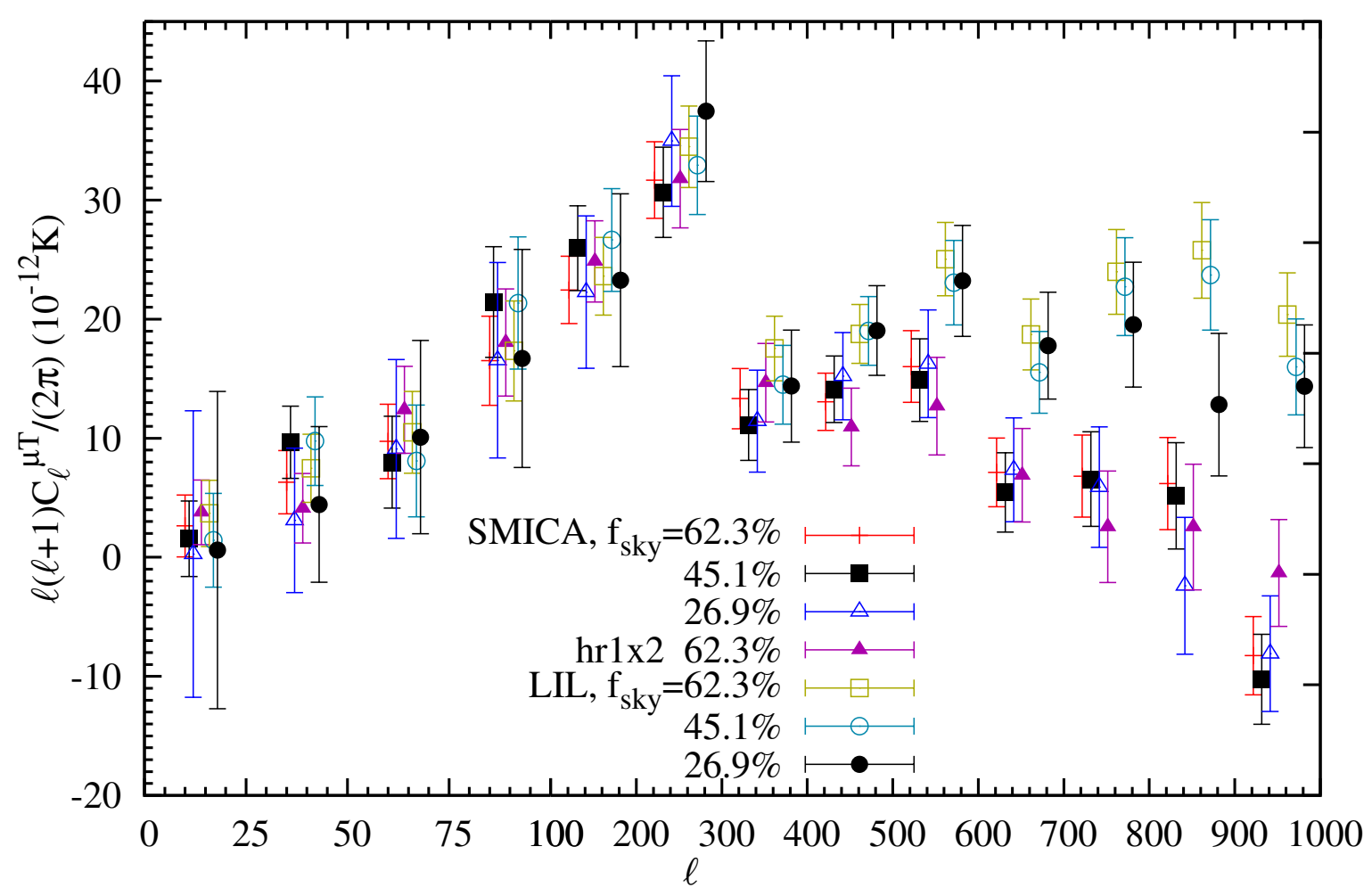

Figure 6. Cross power spectrum of the $\mu$-type distortion fluctuations with CMB temperature anisotropies. The CMB temperature anisotropy maps released by Planck collaboration (SMICA) [45] as well as those created by LIL in [33] have been used. The effects of the effective beams of the respective maps and masks have been corrected for. The points for different masks and map combinations have been offset on the x-axes from the center of the bins to make them distinguishable. At high $\ell$ we use $\Delta \ell=100$ to make the plot less cluttered.

power calculated from the HRHD maps has been subtracted. The power spectrum labeled ' $\mathrm{hr} 1 \mathrm{x} 2$ ' is calculated using the first half-ring $\mu$-map and second half-ring SMICA CMB map. The cross-power spectrum $C_{\ell}^{\mu T}$ is clearly dominated by the residual primary CMB anisotropies. This is the same level of the signal which is also seen if we cross correlate NILC $y$-distortion map [46] and the SMICA CMB map. Thus it seems that for Planck we are limited not by the sensitivity but by the number of channels, the latter limiting our ability to separate the spectral distortions from the primary CMB. The SMICA map is much less noisier and less contaminated on large scales because they use more channels than the 4 channels used by LIL and we use the cross spectrum with SMICA for $62.3 \%$ of sky using full channel data to get the limit

$$
\ell(\ell+1) C_{\ell}^{\mu T} /\left.(2 \pi)\right|_{\ell=2-26}<(2.6 \pm 2.6) \times 10^{-12} \mathrm{~K}
$$

We note that on the large scales all points are consistent while on small scales there is positive correlation between the LIL-CMB and $\mu$ maps but negative correlation between the SMICA CMB and $\mu$ maps. This is just the result of different component separation strategies, in particular the use of $\ell$-dependent component separation in SMICA. 


\section{Theory of fluctuations of $\mu$-type distortions from Silk damping}

The possibility of constraining the primordial non-Gaussianity using the fluctuations of the $\mu$-type distortions was first presented by [26]. Since then there has been considerable work exploring this possibility in detail for many different sources of primordial non-Gaussianity including inflationary models with primordial magnetic fields and isocurvature perturbations [47-54]. We will follow a new approach to the $\mu$-distortion fluctuations from Silk damping motivated by the interpretation of the Silk damping of acoustic modes as mixing of blackbodies [24, 25]. This approach allows for a simple but precise treatment of the $\mu$-distortion fluctuations and obviates the need for 'averaging over an oscillation' and window functions to localize the dissipated energy. We will work in conformal Newtonian gauge [e.g. 55] following the conventions for the metric perturbations as defined in [56].

In the primordial plasma before recombination, photons and baryons are tightly coupled together through Thomson scattering. The mean free path of photons is very small but they still diffuse to lengths much larger than the mean free path by doing a random walk in a sea of electrons. The primordial energy density fluctuations therefore are washed out on this diffusion scale, denoted with comoving wavenumber $k_{\mathrm{D}}(\eta)$, and this process is known as Silk damping [18]. Since the primordial density fluctuations locally had a Planck spectrum because of the thermalization at $z>2 \times 10^{6}$ [9-11], the photon diffusion locally just mixes the photons from different blackbodies. The immediate result of the mixing of blackbodies is $y$-type distortion [57] which rapidly relaxes to equilibrium Bose-Einstein spectrum at $z \gtrsim z_{\mu}=5 \times 10^{4}[9,10,15,17,58-60]$. At smaller redshifts the spectrum remains a $y$-distortion spectrum. The change from $y$-type distortion to $\mu$-type distortion is in reality not abrupt but gradual through an epoch of intermediate-type (or $i$-type) distortions $[15,17,58,60]$ where the initial $y$-type spectrum has relaxed only partially towards the equilibrium Bose-Einstein distribution. We have chosen $z_{\mu}$ to be near the center of the intermediate-type region and will ignore the $i$-type distortions for simplicity as we do not expect them to affect the constraints from Planck. The dissipation of primordial fluctuations not only adds energy but also the photons to the monopole component of the radiation. The $\mu$-distortion created is then given by the total energy dissipated minus the correction from adding photons $[9,10]$

$$
\mu=1.4\left(\frac{\Delta E}{E}-\frac{4}{3} \frac{\Delta N}{N}\right)
$$

where $\Delta E / E$ is the fractional change in the photon energy density and $\Delta N / N$ is the fractional change in the photon number density. This simple equation is just the result of fitting two parameters of the Bose-Einstein spectrum, temperature and chemical potential, to two constraints, the given energy density and number density of photons.

For Silk damping the above equation implies that [24, 25]

$$
\frac{\mathrm{d} \mu(\mathbf{x})}{\mathrm{d} \eta}=-2.8 \frac{\mathrm{d}}{\mathrm{d} \eta}\left\langle\left(\frac{\Delta T(\mathbf{x}, \hat{\mathbf{n}})}{T}\right)^{2}\right\rangle,
$$

where $\mathbf{x}$ is the comoving position coordinates, $\eta$ is conformal time, and $\frac{\Delta T(\mathbf{x}, \hat{\mathbf{n}})}{T}$ is the CMB anisotropy at position $\mathbf{x}$ in direction $\hat{\mathbf{n}}$. We will use bold symbols for vectors with ${ }^{\wedge}$ denoting a unit vector and the same symbol in normal font indicating the amplitude of the vector. Note that $\mathbf{x}$ and $\hat{\mathbf{n}}$ are independent variables (i.e. independent of each other) and describe the 5 of the coordinates in 6-dimensional position-momentum phase space. All terms are functions of $\eta$ which we will suppress for simplicity unless necessary for clarity. Note that the second term in Eq. 5.1 has resulted only in $1 / 3$ of the energy density in the first term going to the $\mu$-type distortion [24, 25]. The angular brackets represent 
an appropriate average that we will describe now precisely. This average, according to our formalism, should lead to mixing of blackbodies locally.

The acoustic dissipation is represented in the Boltzmann hierarchy by the non-vanishing of the photon quadrupole, even in the tight coupling limit [see e.g. 56, 61]. This can be understood as follows. The photons diffusing from different regions/direction to a particular position coordinate have different temperatures. The electrons therefore see non-zero anisotropies and Compton scattering isotropizes the radiation field seen by the electron suppressing the anisotropies. Higher the multipole order more strongly it is suppressed. The largest anisotropy therefore resides in the quadrupole and its dissipation in hydrodynamic terms can be understood as the action of shear viscosity [25]. The mixing of blackbodies by Compton scattering is therefore locally just an angular average and this is precisely what the angular brackets in Eq. 5.2 represent. Taking the Fourier transform of Eq. 5.2 and performing the angular average we get,

$$
\frac{\mathrm{d} \mu(\mathbf{k})}{\mathrm{d} \eta}=-2.8 \frac{\mathrm{d}}{\mathrm{d} \eta} \int \frac{\mathrm{d}^{3} \mathbf{k}^{\prime}}{(2 \pi)^{3}} \int \frac{\mathrm{d} \hat{\mathbf{n}}}{4 \pi} \Theta\left(\mathbf{k}^{\prime}, \hat{\mathbf{n}}\right) \Theta\left(\mathbf{k}-\mathbf{k}^{\prime}, \hat{\mathbf{n}}\right)
$$

where $\Theta(\mathbf{k}, \hat{\mathbf{n}})$ is the Fourier transform of temperature anisotropy and contains a stochastic part coming from the initial curvature perturbations, $\mathcal{R}(\mathbf{k})$, and a deterministic transfer function which we will denote in harmonic space by $\Theta_{\ell}(k)$. The product of anisotropies in real space gives the convolution in Fourier space. We decompose the anisotropies into spherical harmonic coefficients, utilizing the fact that at linear order the transfer functions depend only on the scalar product $\hat{\mathbf{k}} . \hat{\mathbf{n}}$.

$$
\Theta(\mathbf{k}, \hat{\mathbf{n}}) \equiv \sum_{\ell}(-i)^{\ell}(2 \ell+1) \mathcal{P}_{\ell}(\hat{\mathbf{n}} . \hat{\mathbf{k}}) \Theta_{\ell}(k) \mathcal{R}(\mathbf{k}),
$$

where $\mathcal{P}_{\ell}$ are the Legendre polynomials. Substituting in Eq. 5.3 and integrating over $\hat{\mathbf{n}}$ we get

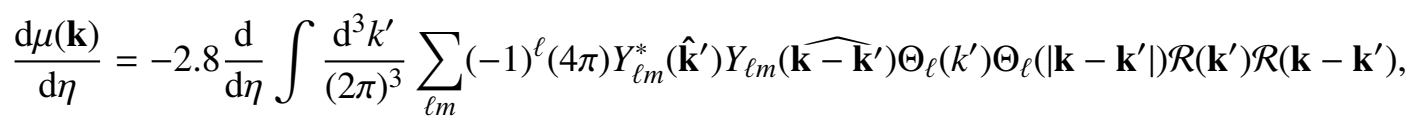

where we have used the addition theorem for spherical harmonics,

$$
\mathcal{P}_{\ell}(\hat{\mathbf{k}} . \hat{\mathbf{n}})=\frac{4 \pi}{2 \ell+1} \sum_{m} Y_{\ell m}^{*}(\hat{\mathbf{k}}) Y_{\ell m}(\hat{\mathbf{n}})
$$

Since we are explicitly doing the angular average of CMB anisotropies locally at position $\mathbf{x}$, our energy dissipation is localized by construction and we do not need a window function to localize the dissipated energy.

In the tight coupling limit almost all of the energy is in the monopole and dipole. The tight coupling solution for the linear CMB transfer functions is [3, 19, 55, 62-64]

$$
\begin{aligned}
& \Theta_{0} \approx A^{i}\left(-3-0.4 R_{v}\right) \cos \left(k r_{\mathrm{s}}\right) e^{-k^{2} / k_{\mathrm{D}}^{2}} \\
& \Theta_{1} \approx A^{i} \frac{\left(-3-0.4 R_{\nu}\right)}{\sqrt{3}} \sin \left(k r_{\mathrm{s}}\right) e^{-k^{2} / k_{\mathrm{D}}^{2}} \\
& \Theta_{\ell} \approx 0 \text { if } \ell \geq 2 \\
& A^{i} \equiv \frac{0.5}{0.4 R_{v}+1.5}=0.3
\end{aligned}
$$

where $r_{\mathrm{s}}(\eta)=\int_{0}^{\eta} \mathrm{d} \eta c_{\mathrm{s}}$ is the sound horizon, $c_{\mathrm{s}}$ is the sound speed of baryon-photon fluid which at $z \gtrsim 5 \times 10^{4}$ is well approximated by $c_{\mathrm{s}} \approx 1 / \sqrt{3}, k_{\mathrm{D}}(\eta)$ is the damping wave number [18-20]. These 
analytic solutions assume that the two metric perturbations $\phi$ and $\psi$ have the same transfer function in the radiation dominated era,

$$
(\phi, \psi)=3\left(\phi^{i}, \psi^{i}\right)\left(\frac{\sin (k \eta / \sqrt{3})-(k \eta / \sqrt{3}) \cos (k \eta / \sqrt{3})}{(k \eta / \sqrt{3})^{3}}\right),
$$

where ${ }^{i}$ indicates the initial perturbation on super horizon scales in the radiation dominated epoch but after neutrino decoupling. These solutions take into account the effect of neutrino anisotropic stress in the initial conditions, $\phi^{i}=-\left(1+0.4 R_{v}\right) \psi^{i}, \Theta_{0}^{i}=-\psi^{i} / 2, \psi^{i}=\mathcal{R} /\left(0.4 R_{v}+1.5\right)$, where $R_{v}=\rho_{v} /\left(\rho_{\gamma}+\right.$ $\left.\rho_{v}\right) \approx 0.409$ is the fraction of neutrino energy density with respect to the total (photons+neutrino) energy density in the radiation dominated epoch after the electron-positron annihilation. At the level of accuracy needed for us, we can set $3+0.4 R_{v} \approx 3$.

Substituting the tight coupling solutions into Eq. 5.5 and taking the ensemble average of the stochastic part, $\mathcal{R}$ terms, we recover the results of $[24,25]$. It is interesting to note that the sum of monopole and dipole terms gives a term $\propto \cos ^{2}\left(k r_{\mathrm{s}}\right)+\sin ^{2}\left(k r_{\mathrm{s}}\right)=1$, i.e. the total energy in the acoustic oscillations adds up to a non-oscillating constant. This is as expected since the sound waves just represent the oscillation of internal energy $\left(\Theta_{0}\right)$ and kinetic energy $\left(\Theta_{1}\right)$ into each other while the total energy of the sound wave must be conserved in the lab frame (at rest with respect to the bulk of the fluid). The energy (and therefore the above interpretation) is of course a gauge or reference frame dependent quantity and we may interpret the above solutions differently in a different gauge [e.g. 65]. In our formalism there is no need to do the average over an oscillation period of the sound wave as the total contribution to the $\mu$-type distortion does not oscillate.

Since we are interested in fluctuations of $\mu$, we will defer the statistical or ensemble average of the stochastic component, $\mathcal{R}$, until the end when we calculate the angular power spectra. At high redshifts, $z \gtrsim 2 \times 10^{6}$, the $\mu$-type distortions are suppressed as the photon creation by double Compton scattering and bremsstrahlung together with comptonization is able relax the spectrum to Planck spectrum, $\mu \longrightarrow 0$. We can take this into account by multiplying the $\mu$ distortion by the blackbody visibility function [9-11], $\mathcal{G}(\eta)$. Integration up to $z=z_{\mu} \approx 5 \times 10^{4}$ then gives us the $\mu$-type distortions at $z_{\mu}$, or the corresponding conformal time $\eta_{\mu}$,

$$
\begin{aligned}
\mu\left(\mathbf{k}, \eta_{\mu}\right)= & -2.8 \int_{0}^{\eta_{\mu}} \mathrm{d} \eta \mathcal{G}(\eta) \frac{\mathrm{d}}{\mathrm{d} \eta} \int \frac{\mathrm{d}^{3} k^{\prime}}{(2 \pi)^{3}} \sum_{\ell m}(-1)^{\ell}(4 \pi) Y_{\ell m}^{*}\left(\hat{\mathbf{k}}^{\prime}\right) Y_{\ell m}\left(\widehat{\mathbf{k}-\mathbf{k}^{\prime}}\right) \\
& \Theta_{\ell}\left(k^{\prime}, \eta\right) \Theta_{\ell}\left(\left|\mathbf{k}-\mathbf{k}^{\prime}\right|, \eta\right) \mathcal{R}\left(\mathbf{k}^{\prime}\right) \mathcal{R}\left(\mathbf{k}-\mathbf{k}^{\prime}\right) .
\end{aligned}
$$

Precise expressions for $\mathcal{G}$ are given in [10] but it is well approximated by the simple expression, sufficient for our purpose, $\mathcal{G} \approx e^{-\left(z / z_{\mathrm{dC}}\right)^{5 / 2}}$, where $z_{\mathrm{dC}} \approx 2 \times 10^{6}$.

We will be interested in the squeezed limit, where we want to calculate the fluctuations that are much larger than the dissipation scale. The dissipation scale here is given by $k^{\prime}$ and the fluctuation scale by $k$. We can therefore take the limit $k \longrightarrow 0$ in the non-stochastic terms to simplify the above expression,

$$
\begin{aligned}
\mu\left(\mathbf{k}, \eta_{\mu}\right) & \stackrel{k \rightarrow 0}{\approx}-2.8 \int_{0}^{\eta_{\mu}} \mathrm{d} \eta \mathcal{G}(\eta) \frac{\mathrm{d}}{\mathrm{d} \eta} \int \frac{\mathrm{d}^{3} k^{\prime}}{(2 \pi)^{3}} \sum_{\ell}(2 \ell+1) \Theta_{\ell}\left(k^{\prime}, \eta\right) \Theta_{\ell}\left(\left|\mathbf{k}-\mathbf{k}^{\prime}\right|, \eta\right) \mathcal{R}\left(\mathbf{k}^{\prime}\right) \mathcal{R}\left(\mathbf{k}-\mathbf{k}^{\prime}\right) \\
& \approx-2.53 \int_{0}^{\eta_{\mu}} \mathrm{d} \eta \mathcal{G}(\eta) \frac{\mathrm{d}}{\mathrm{d} \eta} \int \frac{\mathrm{d}^{3} k^{\prime}}{(2 \pi)^{3}} e^{-2 k^{\prime 2} / k_{\mathrm{D}}^{2}} \mathcal{R}\left(\mathbf{k}^{\prime}\right) \mathcal{R}\left(\mathbf{k}-\mathbf{k}^{\prime}\right),
\end{aligned}
$$

where we have used the tight coupling solution in the last line where the oscillating part evaluates to a constant in the limit $k \rightarrow 0$. The time dependent part is now very simple and is trivially integrated 
if we approximate $\mathcal{G}$ by a step function that is unity for redshifts in the range $5 \times 10^{4} \equiv z_{\mu} \leq z \leq z_{\mathrm{pl}} \equiv$ $2 \times 10^{6}$ and zero otherwise, yielding a final very simple expression for the $\mu$-distortion fluctuations

$$
\mu\left(\mathbf{k}, \eta_{\mu}\right) \approx 2.53 \int \frac{\mathrm{d}^{3} k^{\prime}}{(2 \pi)^{3}}\left[e^{-2 k^{\prime 2} / k_{\mathrm{D}}\left(z_{\mathrm{p}}\right)^{2}}-e^{-2 k^{\prime 2} / k_{\mathrm{D}}\left(z_{\mu}\right)^{2}}\right] \mathcal{R}\left(\mathbf{k}^{\prime}\right) \mathcal{R}\left(\mathbf{k}-\mathbf{k}^{\prime}\right) .
$$

The fluctuations in $\mu$ represent fluctuations in the energy density of photons and these energy density fluctuations would oscillate in the same way as the primary CMB fluctuations. The $\mu$ parameter that we have defined is however gauge invariant, similar to the dimensionless frequency $x=v / T$ and does not undergo oscillations. To be more specific, the adiabatic part of the radiation transfer function has no effect on fluctuations of $\mu$. The $\mu$ fluctuations are therefore frozen in at $z=z_{\mu}$. The dissipative or Silk damping part of the radiation transfer function would affect the $\mu$-fluctuations and $\mu$-type fluctuations would also dissipate on the diffusion scale which keeps on increasing until the recombination. The radiative transfer of the $\mu$-type fluctuations is therefore very simple, it is modified by Silk damping in the same way as the primary CMB anisotropies until recombination and after recombination they just free stream to us. We can take into account the Silk damping very simply to get the $\mu$-distortion fluctuations at the last scattering surface (LSS), $z \approx 1100$,

$$
\mu\left(\mathbf{k}, \eta_{L S S}\right)=\mu\left(\mathbf{k}, \eta_{\mu}\right) e^{-k^{2} / k_{\mathrm{D}}\left(\eta_{\mathrm{LSS}}\right)^{2}}
$$

where $k_{\mathrm{D}}\left(\eta_{\mathrm{LSS}}\right)$ is the damping wavenumber at the last scattering surface which we can evaluate numerically for standard recombination history from the well known expression [18-20, 56, 61]. It is interesting to note that the dissipation of $\mu$ fluctuations will result in mixing of Bose-Einstein spectra giving a $y^{\mathrm{BE}}$-type distortion different from the $y$-type distortion coming from the mixing of Planck spectra. We can of course trivially calculate this distortion using the same formalism used for mixing of blackbodies. For fluctuations $\delta_{\mu}$ around a mean $\bar{\mu}$, we get after mixing a distortion $n_{y}$ BE given by

$$
n_{y^{\mathrm{BE}}}=\frac{1}{2} \frac{\bar{\mu}^{2}\left\langle\delta_{\mu}^{2}\right\rangle e^{x+\bar{\mu}}}{\left(e^{x+\bar{\mu}}-1\right)^{2}}\left(\frac{e^{x+\bar{\mu}}+1}{e^{x+\bar{\mu}}-1}\right),
$$

where we have expanded in Taylor series the spectrum $1 /\left(e^{x+\bar{\mu}\left(1+\delta_{\mu}\right)}-1\right)$. The zeroth order term is just the mean Bose-Einstein spectrum, the first order term vanishes since $\left\langle\delta_{\mu}\right\rangle=0$ by definition and the second order term gives the lowest order distortion. This distortion would be an effect that is 4 th order in perturbations, since $\mu$ is already 2 nd order and we will not pursue this interesting possibility here. Note that the temperature of the Bose-Einstein spectrum would also vary along with the $\mu$ and will give the usual $y$-type distortion at lowest order.

We should note that for modes outside the horizon at the time of recombination, in the SachsWolfe (SW) limit [66], the effect of Silk damping is negligible and we can ignore it,

$$
\mu^{\mathrm{SW}}\left(\mathbf{k}, \eta_{\mathrm{LSS}}\right) \approx \mu\left(\mathbf{k}, \eta_{\mu}\right)
$$

After the recombination the $\mu$-type fluctuations just free stream to us, with a small damping at reionization that we will ignore here, and the equation describing it is just the free streaming Boltzmann equation,

$$
\frac{\partial \mu(\mathbf{k}, \eta)}{\partial \eta}+i k \hat{\mathbf{k}} \cdot \hat{\mathbf{n}} \mu(\mathbf{k}, \eta)=0
$$


Integrating from $\eta_{\text {LSS }}$ to today $\eta_{0}$ we get,

$$
\begin{aligned}
\mu\left(\mathbf{k}, \eta_{0}\right) & =\mu\left(\mathbf{k}, \eta_{\mathrm{LSS}}\right) e^{-i k \hat{\mathbf{k}} . \hat{\mathbf{n}}\left(\eta_{0}-\eta_{\mathrm{LSS}}\right)} \\
& =\mu\left(\mathbf{k}, \eta_{\mathrm{LSS}}\right) 4 \pi \sum_{\ell^{\prime}}(-i)^{\ell^{\prime}} j_{\ell^{\prime}}\left(k\left(\eta_{0}-\eta_{\mathrm{LSS}}\right)\right) \sum_{m^{\prime}} Y_{\ell^{\prime} m^{\prime}}(\hat{\mathbf{n}}) Y_{\ell^{\prime} m^{\prime}}^{*}(\hat{\mathbf{k}}),
\end{aligned}
$$

where we have used the expansion of the exponential function in spherical harmonics [67]. We can now Fourier transform it back to real space and decompose it into spherical harmonic coefficients giving,

$$
\begin{aligned}
\mu_{\ell m}(\mathbf{x}) & =4 \pi(-i)^{\ell} \int \frac{\mathrm{d}^{3} k}{(2 \pi)^{3}} e^{i \mathbf{k} \cdot \mathbf{x}} j_{\ell}\left(k\left(\eta_{0}-\eta_{\mathrm{LSS}}\right)\right) Y_{\ell m}^{*}(\hat{\mathbf{k}}) \mu\left(\mathbf{k}, \eta_{\mathrm{LSS}}\right) \\
& \approx 10.12 \pi(-i)^{\ell} \int \frac{\mathrm{d}^{3} k}{(2 \pi)^{3}} \frac{\mathrm{d}^{3} k^{\prime}}{(2 \pi)^{3}} e^{i \mathbf{k} \mathbf{x}} j_{\ell}\left(k\left(\eta_{0}-\eta_{\mathrm{LSS}}\right)\right) Y_{\ell m}^{*}(\hat{\mathbf{k}}) \\
& \times\left[e^{-2 k^{\prime 2} / k_{\mathrm{D}}\left(z_{\mathrm{pl}}\right)^{2}}-e^{-2 k^{2} / k_{\mathrm{D}}\left(z_{\mu}\right)^{2}}\right] e^{-k^{2} / k_{\mathrm{D}}\left(\eta_{\mathrm{LSS}}\right)^{2}} \mathcal{R}\left(\mathbf{k}^{\prime}\right) \mathcal{R}\left(\mathbf{k}-\mathbf{k}^{\prime}\right),
\end{aligned}
$$

where $\mathbf{x}$ is the position of the observer. This is our final expression for $\mu$-distortion anisotropies which agrees with the corresponding expression in [26] apart from the trigonometric and the window functions which are not needed in our formalism. The damping factor for $\mu$-type fluctuations, $e^{-k^{2} / k_{\mathrm{D}}\left(\eta_{\mathrm{LSS}}\right)^{2}}$, can be neglected in the Sachs-Wolfe limit but must be included when using modes inside the horizon during recombination $(\ell \gtrsim 100)$. For a statistically homogeneous Universe, such as ours is assumed to be, the statistically averaged quantities would not depend on the position of the observer. The dependence on $\mathbf{x}$ would therefore disappear once we take the statistical average, similar to the calculations of the primary CMB anisotropies. To be specific, once we do the statistical average of two quantities with wavenumbers $\mathbf{k}_{1}$ and $\mathbf{k}_{2}$ as arguments, such as for the calculation of the power spectrum, we will get the factor $\delta_{D}^{3}\left(\mathbf{k}_{1}+\mathbf{k}_{2}\right) e^{i\left(\mathbf{k}_{1}+\mathbf{k}_{2}\right) \cdot \mathbf{x}}$ making the argument of the exponent and hence the dependence on the position of the observer vanish [see e.g. 56]. The free streaming solution therefore just projects the 3-d fluctuations at the last scattering surface onto a sphere in the spherical harmonic basis. This completes our derivation of the evolution of $\mu$-distortion fluctuations and the formulae are completely analogous to those of primary CMB anisotropies if we make the instant recombination approximation and free stream the CMB anisotropies from the LSS to today,

$$
\begin{aligned}
a_{\ell m} & =4 \pi(-i)^{\ell} \int \frac{\mathrm{d}^{3} k}{(2 \pi)^{3}} e^{i \mathbf{k} \cdot \mathbf{x}} Y_{\ell m}^{*}(\hat{\mathbf{k}}) \Theta_{\ell}\left(k, \eta_{0}\right) \mathcal{R}(\mathbf{k}) \\
& \approx 4 \pi(-i)^{\ell} \int \frac{\mathrm{d}^{3} k}{(2 \pi)^{3}} e^{i \mathbf{k} \cdot \mathbf{x}} j_{\ell}\left(k\left(\eta_{0}-\eta_{\mathrm{LSS}}\right)\right) Y_{\ell m}^{*}(\hat{\mathbf{k}}) S\left(k, \eta_{\mathrm{LSS}}\right) \mathcal{R}(\mathbf{k}),
\end{aligned}
$$

where $S\left(k, \eta_{\mathrm{LSS}}\right)$ is the source term that reduces in the Sachs-Wolfe approximation to

$$
S^{\mathrm{SW}}\left(k, \eta_{\mathrm{LSS}}\right)=\Theta_{0}\left(k, \eta_{\mathrm{LSS}}\right)+\psi\left(k, \eta_{\mathrm{LSS}}\right) \approx \frac{\psi\left(k, \eta_{\mathrm{LSS}}\right)}{3} \approx \frac{1}{5}
$$

\subsection{The auto and cross power spectrum of $\mu$-distortion fluctuations}

We will assume the following local model for the primordial curvature perturbations:

$$
\mathcal{R}(\mathbf{x})=\mathcal{R}_{G}(\mathbf{x})+\frac{3}{5} f_{\mathrm{NL}} \mathcal{R}_{G}(\mathbf{x})^{2},
$$

where $\mathcal{R}_{G}$ is a Gaussian random field and $f_{\mathrm{NL}}$ is the non-linear parameter. With this model the crosspower spectrum between $\mu$ and $\mathrm{CMB}$ temperature $C_{\ell}^{\mu T} \propto f_{\mathrm{NL}}$ and the auto power spectrum of $\mu$, $C_{\ell}^{\mu \mu} \propto 9 / 25 f_{\mathrm{NL}}^{2} \equiv \tau_{\mathrm{NL}}$. 
We will denote by the angular brackets the statistical ensemble average from now on. For the cross power spectrum we get

$$
\left\langle a_{\ell m} \mu_{\ell^{\prime} m^{\prime}}\right\rangle=(-1)^{m} \delta_{\ell \ell^{\prime}} \delta_{m-m^{\prime}} C_{\ell}^{\mu T},
$$

where $\delta$ represents the Kronecker delta function and

$$
\begin{aligned}
C_{\ell}^{\mu T}= & \frac{10.12}{\pi^{3}} \frac{3}{5} f_{\mathrm{NL}} \int \mathrm{d} k k^{2} j_{\ell}^{2}\left(k\left(\eta_{0}-\eta_{\mathrm{LSS}}\right)\right) S\left(k, \eta_{\mathrm{LSS}}\right) P_{\mathcal{R}_{G}}(k) \\
& \times \int \mathrm{d} k^{\prime} k^{\prime 2}\left[e^{-2 k^{\prime 2} / k_{\mathrm{D}}\left(z_{\mathrm{pl}}\right)^{2}}-e^{-2 k^{\prime 2} / k_{\mathrm{D}}\left(z_{\mu}\right)^{2}}\right] P_{\mathcal{R}_{G}}\left(k^{\prime}\right),
\end{aligned}
$$

where we have used, taking the limit $k \ll k^{\prime}$,

$$
\left\langle\mathcal{R}\left(\mathbf{k}^{\prime}\right) \mathcal{R}\left(\mathbf{k}-\mathbf{k}^{\prime}\right) \mathcal{R}\left(\mathbf{k}_{2}\right)\right\rangle \approx \frac{6}{5}(2 \pi)^{3} f_{\mathrm{NL}} \delta_{D}^{3}\left(\mathbf{k}+\mathbf{k}_{2}\right)\left[P_{\mathcal{R}_{G}}\left(k^{\prime}\right)^{2}+2 P_{\mathcal{R}_{G}}\left(k^{\prime}\right) P_{\mathcal{R}_{G}}(k)\right] .
$$

The first term in the square brackets gives a term of the form $\int \mathrm{d} k k^{2} j_{\ell}(k x)$ independent of the CMB anisotropies which is formally infinite but in the squeezed limit, $k \rightarrow 0$, should vanish and we discard it including only the second term in expression for the $C_{\ell}^{\mu T}$. The integrals are easy to do for a spectrum of form $P_{\mathcal{R}_{G}}=A_{\mathcal{R}_{G}}\left(2 \pi^{2} / k^{3}\right)\left(k / k_{0}\right)^{n_{\mathrm{s}}-1}$ giving us the final expression,

$$
C_{\ell}^{\mu T} \approx \frac{4.86 \pi f_{\mathrm{NL}} A_{\mathcal{R}_{G}}^{2}\left(\eta_{0}-\eta_{\mathrm{LSS}}\right)^{1-n_{\mathrm{s}}}}{k_{0}^{2 n_{\mathrm{s}}-2}} f_{\ell}\left(n_{\mathrm{s}}\right),
$$

where,

$$
\begin{aligned}
& f_{\ell}\left(n_{\mathrm{s}} \neq 1\right)=\frac{\sqrt{\pi}}{4} \frac{\Gamma\left(\frac{3-n_{\mathrm{s}}}{2}\right) \Gamma\left(\frac{n_{\mathrm{s}}-1+2 \ell}{2}\right)}{\Gamma\left(\frac{4-n_{\mathrm{s}}}{2}\right) \Gamma\left(\frac{5+2 \ell-n_{\mathrm{s}}}{2}\right)}\left[2^{-\left(n_{\mathrm{s}}+1\right) / 2} \Gamma\left(\frac{n_{\mathrm{s}}-1}{2}\right)\left(k_{\mathrm{D}}\left(z_{\mathrm{pl}}\right)^{n_{\mathrm{s}}-1}-k_{\mathrm{D}}\left(z_{\mu}\right)^{n_{\mathrm{s}}-1}\right)\right] \\
& f_{\ell}\left(n_{\mathrm{s}}=1\right)=\frac{1}{2 \ell(\ell+1)} \log \left(\frac{k_{\mathrm{D}}\left(z_{\mathrm{pl}}\right)}{k_{\mathrm{D}}\left(z_{\mu}\right)}\right),
\end{aligned}
$$

where $\Gamma$ is the gamma function.

For the auto power spectrum we get,

$$
\begin{aligned}
C_{\ell}^{\mu \mu}= & \tau_{\mathrm{NL}} \frac{10.12^{2}}{2 \pi^{5}} \int \mathrm{d} k k^{2} j_{\ell}\left(k\left(\eta_{0}-\eta_{\mathrm{LSS}}\right)\right)^{2} P_{\mathcal{R}_{G}}(k) e^{-2 k^{2} / k_{\mathrm{D}}\left(z_{\mathrm{LSS}}\right)^{2}} \\
& \times\left(\int \mathrm{d} k^{\prime} k^{\prime 2}\left[e^{-2 k^{\prime 2} / k_{\mathrm{D}}\left(z_{\mathrm{pl}}\right)^{2}}-e^{-2 k^{\prime 2} / k_{\mathrm{D}}\left(z_{\mu}\right)^{2}}\right] P_{\mathcal{R}_{G}}\left(k^{\prime}\right)\right)^{2},
\end{aligned}
$$

where we have used

$\left\langle\mathcal{R}\left(\mathbf{k}^{\prime}{ }_{1}\right) \mathcal{R}\left(\mathbf{k}_{1}-\mathbf{k}^{\prime}{ }_{1}\right) \mathcal{R}\left(\mathbf{k}^{\prime}{ }_{2}\right) \mathcal{R}\left(\mathbf{k}_{2}-\mathbf{k}_{2}^{\prime}\right)\right\rangle=\frac{36}{25} f_{\mathrm{NL}}^{2}(2 \pi)^{3} \delta_{D}^{3}\left(\mathbf{k}_{1}+\mathbf{k}_{2}\right)\left[4 P_{\mathcal{R}_{G}}\left(k_{1}\right) P_{\mathcal{R}_{G}}\left(k_{1}^{\prime}\right) P_{\mathcal{R}_{G}}\left(k_{2}^{\prime}\right)+f\left(k_{1}^{\prime}, k_{2}^{\prime}\right)\right]$,

where $f\left(k_{1}^{\prime}, k_{2}^{\prime}\right)$ represents permutations which are independent of $k_{1}, k_{2}$ in the squeezed limit, $k_{1}, k_{2} \rightarrow$ 0 and would again result in terms in $C_{\ell}$ of form $\int \mathrm{d} k k^{2} j_{\ell}(k x)$ which should vanish in the limit $k \rightarrow 0$. We have also defined $\tau_{\mathrm{NL}} \equiv 9 / 25 f_{\mathrm{NL}}^{2}$. The integrals are again analytically solvable on large scales for power law initial curvature spectrum giving

$$
C_{\ell}^{\mu \mu}=4 \pi \tau_{\mathrm{NL}} \frac{10.12^{2} A_{\mathcal{R}_{G}}^{3}}{k_{0}^{3\left(n_{\mathrm{s}}-1\right)}}\left(\eta_{0}-\eta_{\mathrm{LSS}}\right)^{1-n_{\mathrm{s}}} g_{\ell}\left(n_{\mathrm{s}}\right),
$$


where

$$
\begin{aligned}
& g_{\ell}\left(n_{\mathrm{s}} \neq 1\right)=\frac{\sqrt{\pi}}{4} \frac{\Gamma\left(\frac{3-n_{\mathrm{s}}}{2}\right) \Gamma\left(\frac{n_{\mathrm{s}}-1+2 \ell}{2}\right)}{\Gamma\left(\frac{4-n_{\mathrm{s}}}{2}\right) \Gamma\left(\frac{5+2 \ell-n_{\mathrm{s}}}{2}\right)}\left[2^{-\left(n_{\mathrm{s}}+1\right) / 2} \Gamma\left(\frac{n_{\mathrm{s}}-1}{2}\right)\left(k_{\mathrm{D}}\left(z_{\mathrm{pl}}\right)^{n_{\mathrm{s}}-1}-k_{\mathrm{D}}\left(z_{\mu}\right)^{n_{\mathrm{s}}-1}\right)\right]^{2} \\
& g_{\ell}\left(n_{\mathrm{s}}=1\right)=\frac{1}{2 \ell(\ell+1)}\left[\log \left(\frac{k_{\mathrm{D}}\left(z_{\mathrm{pl}}\right)}{k_{\mathrm{D}}\left(z_{\mu}\right)}\right)\right]^{2}
\end{aligned}
$$

We should point out that we can choose different parameter values for the primordial power spectrum on large and small scales in the above expressions. One factor of $A_{\mathcal{R}_{G}} / k_{0}^{n_{\mathrm{s}}-1} \times$ the term in square brackets in Eq. 5.25 in the expression for $C_{\ell}^{\mu T}$ corresponds to the small scale perturbations and it is possible to choose different $A_{\mathcal{R}_{G}}, k_{0}, n_{\mathrm{S}}$ for these factors to take into account, for example, running of the spectral index or a break in the power spectrum. The same is true for $\left(A_{\mathcal{R}_{G}} / k_{0}^{n_{\mathrm{s}}-1}\right)^{2} \times$ the term in the square brackets in Eq. 5.29 for the $C_{\ell}^{\mu \mu}$.

\subsection{Constraints on primordial non-Gaussianity from $\mu$-distortion fluctuations}

Using the $\Lambda$ CDM cosmology parameters [5, 68], $n_{\mathrm{s}}=0.965, A_{\mathcal{R}_{G}}=2.1 \times 10^{-9}, k_{\mathrm{D}}\left(z_{\mu}\right)=46 \mathrm{Mpc}^{-1}, k_{\mathrm{D}}\left(z_{\mu}\right)=$ $1.1 \times 10^{4} \mathrm{Mpc}^{-1}, k_{0}=0.05 \mathrm{Mpc}^{-1}, \eta_{0}-\eta_{\mathrm{LSS}}=13.9 \mathrm{Gpc}$, we get on large scales

$$
\begin{aligned}
& \frac{\ell(\ell+1)}{2 \pi} C_{\ell}^{\mu T} \approx 2.4 \times 10^{-17} f_{\mathrm{NL}} \\
& \frac{\ell(\ell+1)}{2 \pi} C_{\ell}^{\mu \mu} \approx 1.7 \times 10^{-23} \tau_{\mathrm{NL}},
\end{aligned}
$$

where we have evaluated the expressions at $\ell=13$, the center of our $\Delta \ell=25$ bin in Planck spectra, and the dependence on $\ell$ on large scales is very mild because $n_{\mathrm{s}}$ is very close to 1 . If we choose $n_{\mathrm{s}}=1$, with the other parameters the same, we get slightly larger signal as expected,

$$
\begin{aligned}
& \frac{\ell(\ell+1)}{2 \pi} C_{\ell}^{\mu T}\left(n_{\mathrm{S}}=1\right)=2.9 \times 10^{-17} f_{\mathrm{NL}} \mathrm{K} \\
& \frac{\ell(\ell+1)}{2 \pi} C_{\ell}^{\mu \mu}\left(n_{\mathrm{S}}=1\right)=2.8 \times 10^{-23} \tau_{\mathrm{NL}}
\end{aligned}
$$

Finally we get the constraints on the non-linear parameters using our measurements of the $\mu$ fluctuations,

$$
\begin{aligned}
f_{\mathrm{NL}} & =\frac{\ell(\ell+1)}{2 \pi} \frac{C_{\ell}^{\mu T}}{2.4 \times 10^{-17}} \\
& <10^{5} \\
\tau_{\mathrm{NL}} & =\frac{\ell(\ell+1)}{2 \pi} \frac{C_{\ell}^{\mu \mu}}{1.7 \times 10^{-23}} \\
& <1.4 \times 10^{11}
\end{aligned}
$$

\section{Conclusions}

We have constructed all sky maps of $\mu$-distortion fluctuations from the publicly available Planck HFI data. Our maps and masks are made publicly available at http://www.mpa-garching.mpg.de/ $\sim$ khatri/muresults/. Since we have limited number of channels in Planck, especially channels not dominated completely by foregrounds, it is not possible to completely separate the $\mu$-type and 
$y$-type distortions and our $\mu$-type distortions maps are dominated by the $y$-distortion contamination. Nevertheless, it is possible to put interesting upper limits on the $\mu$ anisotropy power spectrum and cross spectrum of $\mu$ with CMB temperature anisotropies. We have presented measured angular auto and cross power spectra for $\ell \lesssim 1000$ which should be taken as the upper limits.

Our observational constraints on $C_{\ell}^{\mu \mu}$ and $C_{\ell}^{\mu T}$ allows us to constrain new physics which may be responsible for spatially varying energy release in the early Universe, $5 \times 10^{4} \lesssim z \lesssim 2 \times 10^{6}$. We apply our results to constrain the primordial non-Gaussianity for extremely squeezed configurations, $k_{\mathrm{S}} / k_{\mathrm{L}} \approx 5 \times 10^{4}-10^{7}$, where very few constraints exist at present. Our constraints are much stronger, for the same scales, than the recent constraints obtained by [53] using only the CMB temperature anisotropies. The only other comparable constraints, to our knowledge, come from the considerations of formation of primordial black holes from collapse of primordial fluctuations in the presence of large non-Gaussianity $[69,70]$. Our constraints for the non-linear parameters are $f_{\mathrm{NL}}<10^{5}, \tau_{\mathrm{NL}}<1.4 \times 10^{11}$ implying that the non-Gaussianity on these extremely small scales, is smaller than of order unity. We should note that the mean or average distortion $\langle\mu\rangle$ should be of same order of magnitude as the rms, $\mu_{\mathrm{rms}}$, i.e. a strong non-Gaussianity of local type would also increase the average $\mu$-distortion compared to the case of no non-Gaussianity, other parameters being the same. Thus the constraints from COBE-FIRAS on $\langle\mu\rangle$ also constrain primordial non-Gaussianity. Our constraints using the Planck data are however much stronger and in particular constrain the nonlinear term $f_{\mathrm{NL}} \mathcal{R}_{G}^{2}$ to be not larger than the linear term $\mathcal{R}_{G}$ in the primordial perturbation $\mathcal{R}$. With the future experiments, such as Pixie (Primordial Inflation Explorer) and LiteBIRD (Lite satellite for the studies of B-mode polarization) [71], it will be possible to improve on these limits by many orders of magnitude. However, with Planck satellite mission we have already entered a new era of CMB spectrum cosmology.

\section{Acknowledgments}

We would like to thank Nail Inogamov for discussions on the hydrodynamic aspects of the sound waves, in particular the conservation of total energy. This paper used observations obtained with Planck (http://www.esa.int/Planck), an ESA science mission with instruments and contributions directly funded by ESA Member States, NASA, and Canada. We also acknowledge use of the HEALPix software [37] (http: //healpix. sourceforge.net). This research has made use of "Aladin sky atlas" developed at CDS, Strasbourg Observatory, France [72]. This research has also made use of the SIMBAD database, operated at CDS, Strasbourg, France. RS acknowledges partial support by grant No. 14-22-00271 from the Russian Scientific Foundation.

\section{References}

[1] Y. B. Zeldovich, V. G. Kurt, and R. A. Sunyaev. Recombination of Hydrogen in the Hot Model of the Universe. Zh. Eksp. Teor. Fiz., 55:278, 1968. [ADS].

[2] P. J. E. Peebles. Recombination of the Primeval Plasma. ApJ, 153:1, 1968. [ADS].

[3] R. A. Sunyaev and Y. B. Zeldovich. Small-Scale Fluctuations of Relic Radiation. ApSS, 7:3-19, 1970. [DOI], [ADS].

[4] E. L. Wright, C. L. Bennett, K. Gorski, G. Hinshaw, and G. F. Smoot. Angular Power Spectrum of the Cosmic Microwave Background Anisotropy seen by the COBE DMR. ApJL, 464:L21, 1996. [DOI], [ADS].

[5] C. L. Bennett, D. Larson, J. L. Weiland, N. Jarosik, G. Hinshaw, N. Odegard, K. M. Smith, R. S. Hill, B. Gold, M. Halpern, E. Komatsu, M. R. Nolta, L. Page, D. N. Spergel, E. Wollack, J. Dunkley, 
A. Kogut, M. Limon, S. S. Meyer, G. S. Tucker, and E. L. Wright. Nine-year Wilkinson Microwave Anisotropy Probe (WMAP) Observations: Final Maps and Results. ApJS, 208:20, October 2013. arXiv: 1212.5225, [DOI], [ADS].

[6] Planck collaboration, P. A. R. Ade, N. Aghanim, C. Armitage-Caplan, M. Arnaud, and et al. Planck 2013 results. XV. CMB power spectra and likelihood. arxiv:1303.5075, March 2013. [ADS].

[7] R. Keisler et al. A Measurement of the Damping Tail of the Cosmic Microwave Background Power Spectrum with the South Pole Telescope. ApJ, 743:28, 2011. [DOI], [ADS].

[8] R. Hlozek et al. The Atacama Cosmology Telescope: A Measurement of the Primordial Power Spectrum. ApJ, 749:90, 2012. [DOI], [ADS].

[9] R. A. Sunyaev and Y. B. Zeldovich. The interaction of matter and radiation in the hot model of the Universe, II. ApSS, 7:20-30, 1970. [DOI], [ADS].

[10] R. Khatri and R. A. Sunyaev. Creation of the CMB spectrum: precise analytic solutions for the blackbody photosphere. JCAP, 6:38, 2012. [DOI], [ADS].

[11] L. Danese and G. de Zotti. Double Compton process and the spectrum of the microwave background. $A \mathcal{E} A, 107: 39-42,1982$. [ADS].

[12] L. D. Landau and E. M. Lifshitz. Statistical physics. Part.1. Butterworth-Heinemann, Oxford, 1980.

[13] A. S Kompaneets. The establishment of thermal equilibrium between quanta and electrons. Zh. Eksp. Teor. Fiz., 31:876-875, 1956.

[14] Y. B. Zeldovich and R. A. Sunyaev. The Interaction of Matter and Radiation in a Hot-Model Universe. ApSS, 4:301-316, 1969. [DOI], [ADS].

[15] J. Chluba and R. A. Sunyaev. The evolution of CMB spectral distortions in the early Universe. MNRAS, 419:1294-1314, 2012. [DOI], [ADS].

[16] R. A. Sunyaev and R. Khatri. Unavoidable CMB Spectral Features and Blackbody Photosphere of Our Universe. International Journal of Modern Physics D, 22:30014, June 2013. arXiv: 1302 .6553, [DOI], [ADS].

[17] R. Khatri and R. A. Sunyaev. Beyond y and $\mu$ : the shape of the CMB spectral distortions in the intermediate epoch, $1.5 \times 10^{4} \lesssim z \lesssim 2 \times 10^{5}$. JCAP, 9:16, 2012. [DOI], [ADS].

[18] J. Silk. Cosmic Black-Body Radiation and Galaxy Formation. ApJ, 151:459, 1968. [DOI], [ADS].

[19] P. J. E. Peebles and J. T. Yu. Primeval Adiabatic Perturbation in an Expanding Universe. ApJ, 162:815, 1970. [DOI], [ADS].

[20] N. Kaiser. Small-angle anisotropy of the microwave background radiation in the adiabatic theory. MNRAS, 202:1169-1180, 1983. [ADS].

[21] E. M. Lifshitz. J. Phys. (USSR), 10:116, 1946.

[22] R. A. Daly. Spectral distortions of the microwave background radiation resulting from the damping of pressure waves. ApJ, 371:14-28, 1991. [DOI], [ADS].

[23] W. Hu, D. Scott, and J. Silk. Power spectrum constraints from spectral distortions in the cosmic microwave background. ApJl, 430:L5-L8, 1994. [DOI], [ADS].

[24] J. Chluba, R. Khatri, and R. A. Sunyaev. CMB at $2 \times 2$ order: the dissipation of primordial acoustic waves and the observable part of the associated energy release. MNRAS, 425:1129-1169, 2012. [DOI], [ADS].

[25] R. Khatri, R. A. Sunyaev, and J. Chluba. Mixing of blackbodies: entropy production and dissipation of sound waves in the early Universe. AEFA, 543:A136, 2012. [DOI], [ADS].

[26] E. Pajer and M. Zaldarriaga. New Window on Primordial Non-Gaussianity. Physical Review Letters, 109(2):021302, 2012. [DOI], [ADS]. 
[27] D. J. Fixsen, E. S. Cheng, J. M. Gales, J. C. Mather, R. A. Shafer, and E. L. Wright. The Cosmic Microwave Background Spectrum from the Full COBE FIRAS Data Set. ApJ, 473:576, 1996. [DOI], [ADS].

[28] R. Khatri. Linearized iterative least-squares (LIL): A parameter fitting algorithm for component separation in multifrequency CMB experiments such as Planck. ArXiv e-prints, October 2014. arXiv: 1410.7396, [ADS].

[29] R. Khatri and R. A. Sunyaev. Forecasts for CMB $\mu$ - and $i$-type spectral distortion constraints on the primordial power spectrum on scales $8 \lesssim k \lesssim 10^{4} \mathrm{Mpc}^{-1}$ with the future Pixie-like experiments. ArXiv e-prints, March 2013. arXiv: 1303.7212, [ADS].

[30] J. Chluba and D. Jeong. Teasing bits of information out of the CMB energy spectrum. ArXiv e-prints, June 2013. arXiv: 1306.5751, [ADS].

[31] S. Clesse, B. Garbrecht, and Y. Zhu. Testing Inflation and Curvaton Scenarios with CMB Distortions. ArXiv e-prints, February 2014. arXiv: 1402 .2257, [ADS].

[32] Planck Collaboration, P. A. R. Ade, N. Aghanim, C. Armitage-Caplan, M. Arnaud, M. Ashdown, F. Atrio-Barandela, J. Aumont, C. Baccigalupi, A. J. Banday, and et al. Planck 2013 results. IX. HFI spectral response. ArXiv e-prints, March 2013. arXiv: 1303.5070, [ADS].

[33] R. Khatri. An alternative validation strategy for the Planck cluster catalog and \$y-distortion maps. ArXiv e-prints, May 2015. arXiv: 1505.00778, [ADS].

[34] G. Hurier, J. F. Macías-Pérez, and S. Hildebrandt. MILCA, a modified internal linear combination algorithm to extract astrophysical emissions from multifrequency sky maps. $A \mathcal{E} A, 558: \mathrm{A} 118$, October 2013. arXiv: 1007 . 1149, [DOI], [ADS].

[35] J. Delabrouille, J.-F. Cardoso, M. Le Jeune, M. Betoule, G. Fay, and F. Guilloux. A full sky, low foreground, high resolution CMB map from WMAP. AEFA, 493:835-857, January 2009. arXiv:0807.0773, [DOI], [ADS].

[36] Planck Collaboration, N. Aghanim, M. Arnaud, M. Ashdown, J. Aumont, C. Baccigalupi, A. J. Banday, R. B. Barreiro, J. G. Bartlett, N. Bartolo, and et al. Planck 2015 results. XXII. A map of the thermal Sunyaev-Zeldovich effect. ArXiv e-prints, February 2015. arXiv: 1502 .01596, [ADS].

[37] K. M. Górski, E. Hivon, A. J. Banday, B. D. Wandelt, F. K. Hansen, M. Reinecke, and M. Bartelmann. HEALPix: A Framework for High-Resolution Discretization and Fast Analysis of Data Distributed on the Sphere. ApJ, 622:759-771, April 2005. arXiv: astro-ph/0409513, [DOI], [ADS].

[38] Planck Collaboration, P. A. R. Ade, N. Aghanim, M. Arnaud, M. Ashdown, J. Aumont, C. Baccigalupi, A. J. Banday, R. B. Barreiro, R. Barrena, and et al. Planck 2015 results. XXVII. The Second Planck Catalogue of Sunyaev-Zeldovich Sources. ArXiv e-prints, February 2015. arXiv: 1502 .01598, [ADS].

[39] I. Szapudi, S. Prunet, and S. Colombi. Fast Analysis of Inhomogenous Megapixel Cosmic Microwave Background Maps. ApJL, 561:L11-L14, November 2001. [DOI], [ADS].

[40] G. Chon, A. Challinor, S. Prunet, E. Hivon, and I. Szapudi. Fast estimation of polarization power spectra using correlation functions. MNRAS, 350:914-926, May 2004. arXiv: astro-ph/0303414, [DOI], [ADS].

[41] E. Hivon, K. M. Górski, C. B. Netterfield, B. P. Crill, S. Prunet, and F. Hansen. MASTER of the Cosmic Microwave Background Anisotropy Power Spectrum: A Fast Method for Statistical Analysis of Large and Complex Cosmic Microwave Background Data Sets. ApJ, 567:2-17, March 2002. arXiv: astro-ph/0105302, [DOI], [ADS].

[42] G. Efstathiou. Myths and truths concerning estimation of power spectra: the case for a hybrid estimator. MNRAS, 349:603-626, April 2004. arXiv: astro-ph/0307515, [D0I], [ADS].

[43] A. Challinor and G. Chon. Error analysis of quadratic power spectrum estimates for cosmic microwave background polarization: sampling covariance. MNRAS, 360:509-532, June 2005. arXiv: astro-ph/0410097, [DOI], [ADS]. 
[44] M. Tristram, J. F. Macías-Pérez, C. Renault, and D. Santos. XSPECT, estimation of the angular power spectrum by computing cross-power spectra with analytical error bars. MNRAS, 358:833-842, April 2005. arXiv: astro-ph/0405575, [DOI], [ADS].

[45] Planck Collaboration, R. Adam, P. A. R. Ade, N. Aghanim, M. Arnaud, M. Ashdown, J. Aumont, C. Baccigalupi, A. J. Banday, R. B. Barreiro, and et al. Planck 2015 results. IX. Diffuse component separation: CMB maps. ArXiv e-prints, February 2015. arXiv: 1502 .05956, [ADS].

[46] Planck Collaboration, P. A. R. Ade, N. Aghanim, C. Armitage-Caplan, M. Arnaud, M. Ashdown, F. Atrio-Barandela, J. Aumont, C. Baccigalupi, A. J. Banday, and et al. Planck 2013 results. XXI. Cosmology with the all-sky Planck Compton parameter \$y\$-map. ArXiv e-prints, March 2013. arXiv: 1303.5081, [ADS].

[47] J. Ganc and E. Komatsu. Scale-dependent bias of galaxies and $\mu$-type distortion of the cosmic microwave background spectrum from single-field inflation with a modified initial state. Phys.Rev.D, 86(2):023518, 2012. [DOI], [ADS].

[48] M. Biagetti, H. Perrier, A. Riotto, and V. Desjacques. Testing the running of non-Gaussianity through the CMB $\mu$-distortion and the halo bias. Phys.Rev.D, 87(6):063521, March 2013. arXiv:1301.2771, [DOI], [ADS].

[49] K. Miyamoto, T. Sekiguchi, H. Tashiro, and S. Yokoyama. CMB distortion anisotropies due to the decay of primordial magnetic fields. Phys.Rev.D, 89(6):063508, March 2014. arXiv: 1310.3886, [DOI], [ADS].

[50] J. Ganc and M. S. Sloth. Probing correlations of early magnetic fields using $\mu$-distortion. JCAP, 8:18, August 2014. arXiv: 1404.5957, [DOI], [ADS].

[51] A. Ota, T. Sekiguchi, Y. Tada, and S. Yokoyama. Anisotropic CMB distortions from non-Gaussian isocurvature perturbations. JCAP, 3:13, March 2015. arXiv: 1412.4517, [DOI], [ADS].

[52] R. Emami, E. Dimastrogiovanni, J. Chluba, and M. Kamionkowski. Probing the scale dependence of non-Gaussianity with spectral distortions of the cosmic microwave background. ArXiv e-prints, April 2015. arXiv: 1504.00675, [ADS].

[53] A. Naruko, A. Ota, and M. Yamaguchi. Probing small-scale non-Gaussianity from anisotropies in acoustic reheating. ArXiv e-prints, March 2015. arXiv: 1503.03722, [ADS].

[54] M. Shiraishi, M. Liguori, N. Bartolo, and S. Matarrese. Measuring primordial anisotropic correlators with CMB spectral distortions. ArXiv e-prints, June 2015. arXiv: 1506.06670, [ADS].

[55] C.-P. Ma and E. Bertschinger. Cosmological Perturbation Theory in the Synchronous and Conformal Newtonian Gauges. ApJ, 455:7-+, December 1995. arXiv: astro-ph/9506072, [DOI], [ADS].

[56] S. Dodelson. Modern cosmology. Academic Press, Amsterdam, 2003. [ADS].

[57] Y. B. Zeldovich, A. F. Illarionov, and R. A. Sunyaev. The Effect of Energy Release on the Emission Spectrum in a Hot Universe. Soviet Journal of Experimental and Theoretical Physics, 35:643, 1972. [ADS].

[58] A. F. Illarionov and R. A. Sunyaev. Comptonization, characteristic radiation spectra, and thermal balance of low-density plasma. Soviet Astronomy, 18:413-419, 1975. [ADS].

[59] A. F. Illarionov and R. A. Sunyaev. Comptonization, the background-radiation spectrum, and the thermal history of the universe. Soviet Astronomy, 18:691-699, 1975. [ADS].

[60] C. Burigana, L. Danese, and G. de Zotti. Formation and evolution of early distortions of the microwave background spectrum - A numerical study. AEAA, 246:49-58, 1991. [ADS].

[61] S. Weinberg. Cosmology. Oxford University Press, Oxford, 2008.

[62] A. G. Doroshkevich, Y. B. Zeldovich, and R. A. Sunyaev. Fluctuations of the microwave background radiation in the adiabatic and entropic theories of galaxy formation. Soviet Astronomy, 22:523-528, October 1978. [ADS]. 
[63] U. Seljak. A two-fluid approximation for calculating the cosmic microwave background anisotropies. ApJL, 435:L87-L90, November 1994. arXiv: astro-ph/9406050, [DOI], [ADS].

[64] W. Hu and N. Sugiyama. Anisotropies in the cosmic microwave background: an analytic approach. ApJ, 444:489-506, May 1995. arXiv: astro-ph/9407093, [DOI], [ADS].

[65] E. Pajer and M. Zaldarriaga. A Hydrodynamical Approach to CMB mu-distortions. arxiv:1206.4479, 2012. [ADS].

[66] R. K. Sachs and A. M. Wolfe. Perturbations of a Cosmological Model and Angular Variations of the Microwave Background. ApJ, 147:73, January 1967. [DOI], [ADS].

[67] D. A. Varshalovich, A. N. Moskalev and V. K. Khersonskii. Quantum theory of angular momentum. World scientific, Singapore, 1988.

[68] Planck Collaboration, P. A. R. Ade, N. Aghanim, M. Arnaud, M. Ashdown, J. Aumont, C. Baccigalupi, A. J. Banday, R. B. Barreiro, J. G. Bartlett, and et al. Planck 2015 results. XIII. Cosmological parameters. ArXiv e-prints, February 2015. arXiv: 1502 .01589, [ADS].

[69] A. S. Josan, A. M. Green, and K. A. Malik. Generalized constraints on the curvature perturbation from primordial black holes. Phys.Rev.D, 79(10):103520, May 2009. arXiv:0903.3184, [DOI], [ADS].

[70] C. T. Byrnes, E. J. Copeland, and A. M. Green. Primordial black holes as a tool for constraining non-Gaussianity. Phys.Rev.D, 86(4):043512, August 2012. arXiv: 1206.4188, [DOI], [ADS].

[71] M. Hazumi et al. LiteBIRD: a small satellite for the study of B-mode polarization and inflation from cosmic background radiation detection. volume 8442 of Society of Photo-Optical Instrumentation Engineers (SPIE) Conference Series, page 844219, September 2012. [DOI], [ADS].

[72] F. Bonnarel, P. Fernique, O. Bienaymé, D. Egret, F. Genova, M. Louys, F. Ochsenbein, M. Wenger, and J. G. Bartlett. The ALADIN interactive sky atlas. A reference tool for identification of astronomical sources. AEA Supp., 143:33-40, April 2000. [DOI], [ADS]. 